\title{
On Steel Surface Modification via Embedment of ZnNPs@polystyrene Composite as Anti-corrosive Template
}

Ahmed M.A. El Naggar

Egyptian Petroleum Research Institute

Mohamed I Abd el Karim

Nuclear Materials Authority

Mohamed H. Taha

Egyptian Petroleum Research Institute

Ahmed M. Ramadan

Helwan University Faculty of Science

Hanan B.Ahmed ( $\square$ hananbasiony@gmail.com )

faculty of science -helwan university

\section{Research Article}

Keywords: ZnNPs, ZnNPs@polystyrene, Anti-corrosive, Thermodynamic

Posted Date: May 20th, 2021

DOI: https://doi.org/10.21203/rs.3.rs-532908/v1

License: (a) (i) This work is licensed under a Creative Commons Attribution 4.0 International License. Read Full License 


\title{
On Steel Surface Modification via Embedment of ZnNPs@polystyrene Composite as Anti-Corrosive Template
}

\author{
Ahmed M.A. El Naggar ${ }^{1}$, Mohamed I Abd el Karim¹, Mohamed H. Taha², Ahmed M. \\ Ramadan ${ }^{3}$, Hanan B. Ahmed ${ }^{3 *}$ \\ ${ }^{1}$ Egyptian Petroleum Research Institute (EPRI), Nasr city, 11727, Cairo- Egypt \\ 2 Nuclear Materials Authority (NMA) \\ ${ }^{3}$ Chemistry Department, Faculty of Science, Helwan University, Ain-Helwan, Cairo 11795, Egypt \\ * Corresponding author.
} Tel.:+201097411189, E-mail address: hananbasiony@gmail.com (Hanan B. Ahmed)

\begin{abstract}
Corrosion is one of the serious problems countered in different industries as it dramatically causes strong impacting on the infrastructures. As an attempt for sorting out such problem, this approach investigated an innovative strategy for synthesis of corrosion inhibitor for steel surfaces based on composite of polystyrene and zinc nanoparticles (ZnNPs@polystyrene composite). The successive immobilization of ZnNPs (size average of 4-50 nm) within polystyrene matrix (Mwt of $372.587 \mathrm{~g} /$ mole and degree of polymerization equals 3582 repeat unit/ molecule) for clustering of the desirable ZnNPs@polystyrene composite (surface area of $33.62 \mathrm{~m}^{2} / \mathrm{g}$, average pore diameter of $9 \mathrm{~nm}$ ) was approved via several instrumental analyses of FT-IR, XRD, SEM, HRSEM, EDX, TEM and BET with estimation of total pore volume and average pore diameter for the prepared composite. Thermal stability of the prepared composite was affirmed via TGA analysis. Corrosion percentage via weight loss percent in three media of water, $\mathrm{H}_{2} \mathrm{SO}_{4}$ and diesel fuel was estimated to reach maximally to $25 \%$ in case of $\mathrm{H}_{2} \mathrm{SO}_{4}$, while, corrosion inhibition efficiency (CIE) percentage estimated according to weight loss to reach $94.27,88.18$ and $85.05 \%$ after 10 days of soaking the steel samples coated with the synthesized ZnNPs@polystyrene composite (800 ppm) at 25 ${ }^{\circ} \mathrm{C}$, while, elevation of temperature up to $45{ }^{\circ} \mathrm{C}$, resulted in non-significant effect on the estimated CIE to be diminished to $91.8,85.2$ and $81.1 \%$ after soaking in water, diesel fuel and sulfuric acid as corrosion media, respectively. CIE was estimated to be near $30 \%$ in case of coating steel samples with polystyrene polymer, to be significantly increased to near $80 \%$ and formidably jumped to near $100 \%$ by coating with ZnNPs@ @olystyrene composite, with weight percent of $30 \& 50 \%$ of ZnNPs, respectively. In addition to, Zeta potential was also detected to be -9.67 in case of untreated steel samples, while, it became - 4.98 after coating of sample
\end{abstract}


with ZnNPs@polystyrene composite. Eventually, from Arrhenius plots, activation energies and thermodynamic parameters of rate constant, enthalpy $(\Delta H)$ and entropy $(\Delta S)$ confirmed that the interaction is more taking place between the corrosion species and ZnNPs@polystyrene composite as corrosion inhibitor rather than with steel surface. Postulation of the reaction mechanism for the anticorrosive action of the synthesized ZnNPs@polystyrene composite was presented according to the illustrated instrumental analyses.

Keywords: ZnNPs, ZnNPs@polystyrene, Anti-corrosive, Thermodynamic.

\section{Introduction}

Manufacturing and applications of various organic nanocoatings is progressed towards implementation of organic nanocoatings in many industrial purposes owing to their superior capabilities in different fields. Organic nanocoatings are coatings that either has constituents in the nanoscale, or is composed of layers that are less than $100 \mathrm{~nm}$. The small sizes of nanostructures and the high densities of their ground boundaries effectively enable excellent adhesion and good physical coverages of the coated surfaces. Organic nanocoatings were reported to be advantageous with different properties such as surface hardening, adhesive strengthening, long-term and/or high-temperature corrosion resisting, etc. In addition to, organic nanocoatings can be exploited as thinner and smoother thickness, that could allow the flexibility in equipment designing, enhanced the efficiency, lowering the fuel cost effectiveness, lowering carbon footprints, and lowering the maintenance and operating costing. Organic nanocoatings were also utilized superiorly in the reduction of the effectiveness of a corrosive environment.

Corrosion inhibition could be achieved via several techniques, while, the selection of the right methodology must be preciously performed. Optimization between the cost effectiveness, the performance, and the corrosion effectiveness must be also taken in account. Corrosion could be inhibited via considering some of key factors as follows; firstly, the choice of material with anticorrosive action, where the material must be either nonreactive within galvanic series or could give a anticorrosive oxide layer; secondly, controlling the environmental conditions, such like, adding the inhibitors (Lamaka et al. 2007; Rahmani et al. 2016), controlling $\mathrm{pH}$ and the temperature of the surrounding medium, reduction in oxygen (Cho et al. 2014), sulphur, and chloride contents (Du et al. 2016), diminishing velocity of flowing, cleaning up from sediments, etc.; thirdly, the surface modification, that could be achieved via uploading of the physical barriers like films and coatings in order to decrease the 
crevices and cracks (Calderón et al. 2016; Mária 2014); in addition to, cathodic protection, in which the corrosion currents could be suppressed and is pushed for metal protection, that could be realized via power source or via connecting with more reactive (anodic) materials to the protected surface (Wang et al. 2015).

According to literature, there are three postulated mechanism of corrosion inhibition action that could be briefly presented in the following points (Dariva and Galio 2014; Lallam 2015); i) adsorption: in which the anticorrosive reagent could be chemically adsorbed on the surface of the treated steel to form a protective thin layer with corrosion inhibition effects; ii) surface layer via forming of oxide membrane for protecting the metallic surfaces; iii) passivation in which the inhibitors could react with corrosive species in surrounding media, to form a protective precipitate.

Special methodologies were reported for synthesis of polymer nanocomposites to be applicable for protection of metallic surface against corrosion such like, wet-chemical reduction, photochemical reduction, microwave assisted methodology, laser mediated technique hydrothermal technique...etc... However, all the previously-mentioned techniques could be divided into only two groups of techniques i.e. ex-situ and in-situ methodologies. In ex-situ techniques, all methods could be encompassed while the nanoparticles are in-grained outside the host polymeric matrices. On the other hand, pre-growing of the nano-sized particles could be directly proceeded within the polymeric matrices is known as in-situ technique (Salehoon et al. 2017). Ex-situ technique is advantageous with promotion of accurate controlling of size and geometrical shaping, in addition to being an approachable technique for industrial-scale application (Quadri et al. 2017). On the other hand, in-situ technique is reported to be mostly done in single-pot approach, while, ingraining of nanoparticles among the host matrix inhibits their agglomeration for achieving the desirable spatial dispersion, making this technique numerously applied in researches. However, in-situ technique is disadvantageous with the existence of unreacted constituents with the generated composite (Solomon et al. 2017).

This approach investigated an innovative strategy for synthesis of corrosion inhibitor for steel surfaces based on composite of polystyrene and zinc nanoparticles (ZnNPs@polystyrene composite). The successive immobilization of ZnNPs within polystyrene matrix for clustering of the desirable ZnNPs@polystyrene composite was approved via several instrumental analyses of FT-IR, XRD, SEM, HRSEM, EDX, TEM and 
BET with estimation of total pore volume and average pore diameter for the prepared composite. Thermal stability of the prepared composite was affirmed via TGA analysis. Corrosion percentage via weight loss percent in three media of water, $\mathrm{H}_{2} \mathrm{SO}_{4}$ and diesel fuel was estimated. Corrosion inhibition efficiency (CIE) percentage estimated according to the weight loss for the steel samples coated with the synthesized ZnNPs@polystyrene composite. Effect of temperature elevation on the estimated CIE after soaking in water, diesel fuel and sulfuric acid as corrosion media was also detected. CIE was estimated for coating steel samples with ZnNPs@polystyrene composite with different doses of ZnNPs. Zeta potential was also detected for untreated and ZnNPs@polystyrene composite treated samples. Eventually, from Arrhenius plots, activation energies and thermodynamic parameters of rate constant, enthalpy $(\Delta H)$, entropy $(\Delta S)$ was also evaluated. Postulation of the reaction mechanism for the anticorrosive action of the synthesized ZnNPs@polystyrene composite was presented according to the illustrated instrumental analyses.

\section{Experimental section}

In this research study, a composite structure made of combining polystyrene and zinc nanoparticles is introduced as a novel corrosion inhibitor. All the chemical reagents that were used to prepare either the organic or inorganic parts of the composite are reported in the following section.

\section{Materials}

Unless otherwise stated, all the presented chemical reagents, during this research work, were of analytical grade and they were utilized without any further treatments. Table 1 provides full details of sources and purities of these chemicals reagents.

Table 1

\section{Synthetic methodologies}

This section is covering two main points which are the experimental procedures of preparing the introduced composite (corrosion inhibitor) and its related characterizations. The preparation of ZnNPs@polystyrene composite had included two stages namely; preparation of a porous polystyrene (as a support) and the coating of this support via loading of ZnNPs via electroless platting (ELP) technique.

The polystyrene polymer was prepared using the high internal phase emulsion (HIPE) polymerization technique, as reported in literature (El Naggar et al. 2015). This technique is 
based on combination of an oil phase with another aqueous phase to obtain an emulsion solution. However, the aqueous phase is always presenting the majority of the emulsion content $(70-90 \%)$ in order to produce a polymer with a porous nature. On the other hand, the increase of the oil phase percentage, during the preparation stage, is in turn responsible for elevating the molecular weight of the produced polymer at the end. The mechanism of HIPE technique is based on the dispersion of the aqueous phase molecules in between the oil phase ones to produce micelles in the obtained emulsion solution. These micelles are then transformed to pores along the geometry of the ultimately obtained polymer.

Experimentally, the synthesis of the polystyrene structure had launched via the preparation of the oil phase which included the styrene monomer $(90 \%)$ and $(10 \%)$ of the surfactant, by weight $\%$. On the other hand, the aqueous phase had been simultaneously prepared. The latter phase contained respective weight percentages of $98 \%$ \& $2 \%$ from both de-ionized water and potassium persulfate (as a polymerization initiator). Then, the oil phase was added to the aqueous phase under vigorous stirring at the pre-determined polymerization conditions. Fig. 1a showed a schematic diagram for the process of polystyrene preparation through emulsion polymerization technique.

The practical preparation procedures had started by inserting the specified amount of de-ionized water into a $1000 \mathrm{ml}$ round flask which was then heated up to $60{ }^{\circ} \mathrm{C}$. As soon as the temperature was reached, the initiator and oil phase were consecutively added to the flask. The whole ingredients had been then continuously stirred vigorously (500 rpm) under reflux for a reaction time of two hours. This reaction time, as prior stated in the related literature survey, could be sufficient to convert approximately $90 \%$ of the styrene monomer into polystyrene. After the completion of two hours under reflux, the obtained emulsion solution was then transferred into warm oven (at $65{ }^{\circ} \mathrm{C}$ ) in order to complete the polymerization process. This step was also necessary to attain the stage of aqueous phase decanting.

As soon as the polystyrene was synthesized, it was forwarded to the current stage in which it was used as a support for zinc nanoparticles. Particularly, ZnNPs@polystyrene composite which had contained equal weight percentages $(50: 50)$ of polystyrene and zinc metal was prepared in this stage. This composite was prepared by using the ELP method as reported in (El Naggar et al. 2015). The steps of loading the zinc nanoparticles onto the polystyrene (support) were carried according to the following sequence: 
i. A solution (0.2 M) was prepared by dissolving $5.453 \mathrm{gm}$ of $\mathrm{ZnCl}_{2}$ (which contain $2.616 \mathrm{~g}$ of $\mathrm{Zn}$ metal) in $200 \mathrm{~mL}$ deionized water.

ii. The solution was kept under vigorous stirring (at $500 \mathrm{rpm}$ ) and consequently heated up to $90{ }^{\circ} \mathrm{C}$ until a clear solution was observed.

iii. Equal molar ratio to that of zinc ions was then added from the reducing agent namely; $4.048 \mathrm{~g}$ of $\left(\mathrm{C}_{2} \mathrm{H}_{5}\right)_{3} \mathrm{~N}$, were added to the solution for generation of $\mathrm{ZnNPs}$.

iv. The whole mixture was then allowed to stir under heating for another 10 minutes. Then $2.616 \mathrm{~g}$ of polystyrene, which is equal to the amount of the pre-added $\mathrm{Zn}$ metal, were added to the solution.

v. After the addition of polystyrene, the solution was kept under heating with vigorous stirring for 20 minutes in order to allow the complete interaction between the components to take place. At the end of the reaction time, the stirring was hold and reaction vessel was kept under heating for another 20 minutes, to complete the metallization of polymer particles.

vi. Heating was afterward stopped and the solution was left to settle down for $2 \mathrm{~h}$ to allow all the metalized particles of polymer to segregate at the bottom of the reaction vessel. The composite was ready and was subsequently collected by filtration. Then, it was washed several times by distilled water and was next left to dry, for $12 \mathrm{~h}$, in a desiccator at room temperature (Fig. 1b).

\section{Characterization and instrumental analysis}

The verification of structural characteristics of the prepared powder polymers was studied by Fourier-transform infrared spectroscopy (FTIR) device model Thermo Nicolet Avatar 370, Germany. The molecular weight of the collected polymer was conducted by Gel permeation chromatography (GPC) technique. The surface characteristics of the porous polymer were examined using Brunauer-Emmett-Teller (BET) surface area analyzer model SA 3100, manufactured by A Beckman-Coulter-USA. The surface morphology of polymer was acquired through scanning electron microscope (SEM), model Quanta 250 FEG. This microscope has accelerating voltage of $30 \mathrm{KV}$ and magnification range between 14 and 1000000 times with a high resolution of $1 \mathrm{~nm}$. The SEM device is equipped with energetic dispersive X-ray (EDX) unit to facilitate the quantitative elemental analysis of specimens. 
The verification of structural characteristics of the prepared nano-composite was first carried out by Fourier-transform infrared spectroscopy (FTIR). Then, the structural properties and degree of crystallinity as well as the average crystal size of the prepared nano-composites was illustrated by X-ray diffraction (XRD). Diffractometer model ARL ${ }^{\text {TM }}$ X'TRA $^{\prime T}$ manufactured by Thermo Fisher Scientific, USA was utilized to perform this test. The surface characteristics; including specific surface area, average pores diameter and total pore volume, of ZnNPs@polystyrene composite structure was examined using Brunauer-Emmett-Teller (BET) surface area analysis.

The surface morphology of the composite was acquired through high resolution scanning electron microscopy (HRSEM). Sample mapping and elemental analysis of the introduced composite was performed via the EDX unit which is fitted to the Scanning microscope. Additionally, the produced ZnNPs@polystyrene composite was examined by transmission electron microscope (TEM), model JEOL-2100M, manufactured in Japan, in order to explore its internal morphology. The thermal behavior of the presented composite was acquired by thermal gravimetric analysis (TGA).

After ZnNPs@polystyrene composite was prepared and fully characterized using the proper analytical techniques, it was forwarded to test its efficiency as a solid dispersant corrosion inhibitor. The use of such composite in the solid state form can be counted as a certain novelty of this research work. The efficiency of the prepared composite in preventing the corrosion of carbon steel surfaces was investigated in four different media. Specifically, pure water, formation, diesel fuel and sulfuric acid, were utilized as corrosive media during this study.

A commercial carbon steel sample which has the following chemical composition, $\mathrm{C}$ : $0.21 \%$, Mn: $0.593 \%$, Si: $0.044 \%$, S: $0.032 \%$ and Fe: $98.4 \%$, was utilized during this research work. The choice of this sample had based on the fact that it is the most widely used steel material due to its low cost, availability and their good fabrication qualities. Additionally, such type of steel material found its usage in various reaction vessels such as cooling towers, boilers, pipelines and sections for use in bridges as well as in delivery tanks of different liquid fractions (Senthilkumar and Ajiboye 2012). The as-received carbon steel samples were machined into cuboid shaped test specimens (coupons) of about $10 \mathrm{~mm}$ thickness and length of $50 \mathrm{~mm}$ using a steel cutting machine. Firstly, the coupons were gently polished using a soft polishing sheet. Then, they were sequentially cleaned via using ethanol where they were next rinsed several times by distilled water and were left to dry into a desiccator. 
As previously mentioned, four corrosive media had been selected to assess the efficiency of the introduced ZnNPs@polystyrene composite as a corrosion inhibitor. The choice of these media is of a particular relevance to their presence in various industries. Specifically, sulfuric acid $\left(\mathrm{H}_{2} \mathrm{SO}_{4}\right)$ is one of the chemical compounds that is frequently used in industries alongside the carbon steel components. On the other hand, water and formation water are two of the major media that can be extensively present during the transportation of petroleum crude oils. Additionally, diesel fuels are of the most abundant liquid petroleum fractions that are widely distributed globally as energy sources. Such fractions are usually containing high levels of sulfur compounds which are known of being highly corrosive components. The delivery of either diesel fuels or crude oils is generally carried out through usage of tanks that are made of carbon steel materials. Therefore, testing the efficiency of the introduced corrosion inhibitor, in this research work, in these three media as well as sulfuric acid had been of high importance.

Before evaluating the efficiency of the presented ZnNPs@polystyrene composite in inhibiting the corrosion, it was necessary to perform control experiments in the aforementioned four corrosive media. Through these experiments, the formerly cleaned and dried carbon steel coupons were placed in the corrosive media in a complete absence of the prepared corrosion inhibitor. Particularly, four coupons were placed separately in four beakers; each of them contain $100 \mathrm{~mL}$ of one of the corrosive media. However before inserting the coupons into the test vessels, their weights were determined using a four decimal digits balance. The carbon steel coupons were remained in the corrosive media for a pre-determined time intervals. In particular, 1, 3, 5, 7 and 10 days were set as the time of testing. By the end of each time interval, the carbon steel specimens were picked from the various corrosive media in order to calculate their corrosion amounts. Each of the control experiments had been performed in triplicate to accurately measure the corrosion values that could be attained by each of the corrosive media.

After the control experiments were done, testing the corrosion inhibition efficiency by using the prepared ZnNPs@ polystyrene composite was then carried out. Same procedures, as the former stated in control experiments, had been employed during this stage. However, in each of the vessels, which contain the corrosive media, the corrosion inhibitor was added at certain amounts. Specifically, concentrations equal 200, 400 and $800 \mathrm{ppm}$ of ZnNPs@polystyrene composite were added on separate basis in each of the testing vessel. In this stage, testing the effect of ZnNPs@polystyrene composite dose on the efficiency of inhibiting the corrosion of the carbon steel coupons had been tracked. 
In the previous stage the most proper amount of the corrosion inhibitor was determined and was then applied in the following step of testing. In the second step of testing, the effect of temperature $\left(25,35\right.$ and $\left.45^{\circ} \mathrm{C}\right)$ on the quality of corrosion inhibition efficiency was examined. In each of the latter two steps of testing the effect of each corrosive media on the Inhibition efficiency was investigated on a comparative basis. The detection of corrosion amounts (in grams) of the carbon steel specimens was carried out through the weight loss route. The weight change for each of the specimen was determined in 24 hours, at first place, then it was progressively obtained up to 240 hours. These weight losses were then used to determine the corrosion percentage according to the coming expression:

\section{$\%$ Corrosion $=100-\mathrm{W}_{1} / \mathrm{W}_{\mathrm{o}} \times 100$}

where $W_{o}=$ original weight of carbon steel coupon and $W_{1}=$ weight of the inhibited coupons after a time of testing.

The efficiency of corrosion Inhibition efficiency (\%) could be then evaluated using the following expression:

\section{$\%$ Inhibition $=\Delta \mathbf{W} / \mathbf{W}_{\mathbf{0}} \times 100$}

Where $\Delta W($ weight loss $)=$ initial weight of steel coupon $\left(W_{o}\right)-$ weight after placement in the corrosive media $\left(W_{1}\right)$

After the corrosion amounts and their related percentages of inhibiting the corrosion of the carbon steel coupons via weight loss technique, the steel samples were further subjected to some analytical techniques. Particularly, Zeta potential and contact angle techniques were applied to the collected steel coupons after both the control and corrosion inhibition experiments. This stage was carried out in order to verify the obtained findings through the weight loss calculations. Additionally, these analyses were meant to comparatively explore, in depth, the effect of operating parameters as well as impact of various corrosive media on corrosion inhibition quality.

\section{Results and Discussion}


Corrosion could be defined as the deterioration of metallic surfaces attributing to their interaction with corrosive elements in their surrounding medium, such like $\mathrm{Cl}^{-}, \mathrm{F}^{-}, \mathrm{CO}_{2}, \mathrm{O}_{2}$, etc. Deterioration due to corrosion resulted in losing of materials, damaging of equipment and decrement in the efficiency. Inhibition of corrosion could be performed via various techniques, while, the choice of the right technique must be done taking in account the optimization between the cost, the process performance, and corrosion effectiveness.

Nanocomposites based on transition metals were in limelight owing to their superior applicability in various scientific and technological purposes. Synthesis of zinc based thin membranes could emerge a great consideration, attributing to their mechanical and chemical stabilities. Therefore, zinc based coating layer deposited on the steel specimens is supposed to exhibit high anti-corrosive performance. In addition to, no reports were considered with studying the enhancement of the corrosive resistance for metal surfaces via coating the surface with ZnNPs based composite thin films. In the current study, a new approach is provided through the utilization of nanotechnology concept in production of a solid corrosion inhibitor. Herein, zinc nanoparticles (ZnNPs) was immobilized within polystyrene for preparation of ZnNPs@ polystyrene composite to act as a novel inhibitor for corrosion under the influence of various corrosive media.

Molecular weight of the as-produced polystyrene was acquired via the gel performance chromatography (GPC) technique and it was found to be $372.587 \mathrm{~g} /$ mole. This value is referring to a degree of polymerization equals 3582 repeat unit/ molecule. This increased value of molecular weight and polymerization degree could be attributed to use of an oil phase represents $30 \%$ per weight of the whole composition of the emulsion solution during the polymerization stage. Therefore, the production of long chained polystyrene, having a high molecular weight, could be successfully achieved.

After determining the molecular weight of the acquired polystyrene polymer, it was then metalized by zinc nanoparticles for production of ZnNPs@polystyrene composite using electroless platting technique, which depends on the catalytic reduction of metal ions in an aqueous solution and subsequent deposition of metal without the use of electrical energy. The driving force for both reduction and deposition is accomplished by using a chemical reducing agent. It is also known as autocatalytic plating due to the potential developments of the substrate through the process when it is submerged in electroless solution which contains a source of metallic ions, reducing agent, stabilizer and other components. In the current study, 
zinc chloride was used as metal precursor and triethylamine was exploited as the reducing agent. Zinc ions were reduced to generate zero valent zinc atoms, which were subsequently deposited on the surface of polystyrene macromolecules which currently functionalized as the supporting template or stabilizer for successive ingraining of $\mathrm{Zn}$ nanoparticles and sequential clustering of ZnNPs-polystyrene composite. Zinc nanoparticles could represent $50 \mathrm{Wt}$. \% of the ultimately obtained ZnNPs@ polystyrene composite at this stage. The confirmation of such percentage, of $\mathrm{Zn}$ nanoparticles within the structure of this composite, was acquired through gravimetric measurements during the experimental loading of zinc onto the polymer structure.

\section{Structural Characteristics}

The structural analysis of both the as-prepared polystyrene and the consequently obtained ZnNPs@polystyrene composite had been first discussed through the presented XRD patterns in Figure 2. XRD pattern of the blank polystyrene (Figure 2a) could reveal broad hump peak positioned between $2 \theta$ values of 15 and $30^{\circ}$. The observed XRD signal is referring to the highly amorphous nature of the as-prepared polystyrene polymer (Wadi et al. 2020). A rational change in the XRD pattern could be noticed for ZnNPs@polystyrene composite, as Xray diffractogram could exhibit strong sharp peaks indicative to the zinc metal. Specifically, high intensity Bragg diffraction peaks could be observed at $2 \theta=11.71,17.03,22.26,25.41$, $33.76,34.28$ and $58.44^{\circ}$. The peak of the highest intensity which is shown, in the above diffractogram (Figure 2b), at $2 \theta$ of $11.71^{\circ}$ is indexed as the $d 200$ for zinc metal (Jassim et al. 2016).

The sharpness of the exhibited peaks could reflect the formation of zinc nanoparticles in the form of crystals. The high intensity of the XRD signals is referring to the elevated degree of crystallinity (number of crystalline particles) along the prepared ZnNPs@polystyrene composite. The detection of sharp XRD signals with no observation for strong hump peak, indicative to the polystyrene polymer, could show the complete domination and well dispersion of zinc nanoparticles onto the polymer geometry. Using the provided XRD data in Figure $\mathbf{2 b}$, the mean crystals size of ZnNPs@ polystyrene composite was calculated via Scherer's equation and it was found to be $61 \mathrm{~nm}$.

Figure 2

After confirming the detection of metal-organic structure via XRD patterns, the structural (function) groups of organic structure in the introduced ZnNPs@polystyrene composite, as a corrosion inhibitor, was tracked via FTIR analysis. FTIR spectroscopic study 
of the illustrated ZnNPs@ polystyrene composite has been displayed in Figure 3. In Figure 3a, absorption bands at 3025.40 and $3066 \mathrm{~cm}^{-1}$ referring to both $\mathrm{CH}$ - aliphatic and $\mathrm{CH}$-aromatic could be noticed in the exhibited IR spectrum. Additionally, the presence of $\mathrm{C}=\mathrm{C}$ that belong to benzene ring could be verified through an absorption band at $1596.86 \mathrm{~cm}^{-1}$. Also, bands for both asymmetric and symmetric $\mathrm{CH}_{2}$ could be noticed at 2921.03 and $2853.29 \mathrm{~cm}^{-1}$ respectively (Al-Kadhemy et al. 2016). Furthermore, an absorption band referring to P-substituted benzene ring could be detected at Wavenumber of $720 \mathrm{~cm}^{-1}$. An absorption band at $3449 \mathrm{~cm}^{-1}$, assigned for $\mathrm{OH}$ group, could be also noticed for the polystyrene polymer. The observation of such peak is attributed to the stuck of either some water molecules within the polymer structure, during the polymerization stage or the present $\mathrm{OH}$ in the radicals that could be generated, from potassium persulfate, as an initiator, during the polymer formation (Al-Kadhemy and Rasheed 2013).

The FTIR spectrum of the ZnNPs@polystyrene composite (Figure 3b) had revealed the presence of some additional absorption bands to those the previously stated in Fig. 3a. Particularly, absorption peaks located around wavenumber values of 470,847 and $1100 \mathrm{~cm}^{-1}$ could be detected through the displayed spectrum. These absorption bands are indicative to vibrational zinc particles (Ayeleru et al. 2019). On the other hand, variations in the intensities of the detected absorption bands for polystyrene structure (Figure $\mathbf{3 b}$ ) than those of its reprehensive bands in Figure 3a could be noted. These variations are explained due to the loading of zinc nanoparticles onto the polymer structure (Xu et al. 2012). This observation may consequently verify the increased propagation of ZnNPs along the texture of polystyrene, in the produced ZnNPs@polystyrene composite. No absorption bands referring to a chemical interaction between zinc particles and carbon atoms of polystyrene could be detected. This in turn can clarify that the combination of zinc nanoparticles and polystyrene could take place via stable embedment within intermolecular spaces of polystyrene matrix.

Figure 3

The stability of such embedment was tested through a leaching stage by soaking 200 ppm $(0.2 \mathrm{~g} / \mathrm{L})$ of the prepared ZnNPs@polystyrene composite into two different media (formation water and diesel fuel) for a time of 7 days. The composite particles were then washed several times by double distilled water and had been subsequently dried at $80{ }^{\circ} \mathrm{C}$ for 3 $\mathrm{h}$ inside a vacuum furnace. The dried samples were weighed up and were shown constant weights $(0.2 \mathrm{~g})$. This could consequently reflect that no release (leaching) of zinc nanoparticles, 
from ZnNPs@polystyrene composite, had under taken; hence, a stable embedment could be detected. The stability of embedment can be attributed to the successive entrapping of ZnNPs within the intermolecular spaces between polymeric chains of polystyrene.

\section{Textural Properties}

As soon as the characteristics of the presented structures were explored via both XRD and FTIR analyses, their textural characteristics were then investigated through SEM, HRSEM, EDX and BET surface area techniques as shown in Figure 4 and Table 2 respectively. The formerly presented analyses (XRD and FTIR) had confirmed the inclusion of both the organic (polymer) and inorganic (Zn metal nanoparticles) sites within the structure of the synthesized ZnNPs@polystyrene composite. However in order to clearly find out about the combination of these sites together (as a composite), it was necessary to scan both the blank polystyrene and its ZnNPs@polystyrene composite under the electronic microscope. The exhibited SEM images in Figure 4 illustrate the surface morphology of both the as-prepared polystyrene and its sub-driven composite.

The given SEM images, Figure 4a, show a smooth uniform sheet-like surface. This sheet contains a number of meso-pores, which morphologically is in a strong agreement with the next illustrated surface characteristics. Observable changes in the surface morphology of the polymer could be seen after loading of Zn nanoparticles within its structure, Figures $\mathbf{4 b}$ and 4c. The surface has become a bit wavy and non-smooth while being of a uniform appearance. It can be also seen that all the detected pores in the blank polymer had totally disappeared. The observed change in surface morphology is referred to the stable dispersion and complete domination of tightly porous $\mathrm{Zn}$ nanoparticles along the surface of polystyrene (Tang et al. 2007).

In accordance to Figures 4b and 4c, ZnNPs@polystyrene composite had exhibited well-dispersed crystals of zinc nanoparticles along the whole surfaces of polystyrene. The observed zinc crystals, through both SEM and HRSEM images, are of different shapes. Specifically, tetragonal, pentagonal and hexagonal crystals of metallic zinc could be noted through the presented scanning images. However, the detected Zn particles, despite of being highly dispersed along the polymer structure, could show some accumulations of subsequent layered crystals. This observation can be referred to the over-loading of zinc particles $(50 \%)$ of the ZnNPs@polystyrene composition, by weight during the preparation procedures. In addition, the high percentage of zinc loading was accompanied by the high molecular weight 
of the polymeric structure. This in turn could likely limit the penetration of a number of zinc particles into the polymer geometry. Therefore, some of metallic zinc particles could accumulate onto one another at the surface of support (polystyrene).

The exhibited morphology for ZnNPs@polystyrene composite has been in a good agreement with the previously given XRD pattern, Figure $\mathbf{2 b}$, which could also show complete domination of $\mathrm{Zn}$ particles. The domination of zinc crystals onto the surface of polystyrene is expected to be highly advantageous in its proposed application as a corrosion inhibitor. On the other hand, the observed sizes of zinc particles in the ZnNPs@ polystyrene composite, through SEM images, could confirm the formation of a nanocomposite structure via detection of $\mathrm{Zn}$ particles in the range of nanoscale. In an agreement with this finding, the illustrated TEM images in Figure 4d could verify the presence of a nanocomposite structure. Particularly, particles in the size range of 4-50 nm could be observed at different bits along the morphology of ZnNPs@polystyrene composite. TEM micrographs could additionally exhibit high degree of $\mathrm{Zn}$ particles dispersity around the whole of polymer geometry. Furthermore, four, five and sex sided Zn crystals had been noted through the TEM images. The similarity of both surface and internal morphologies of the ZnNPs@polystyrene composite could reflect the high uniformity that could be achieved during its preparation procedures.

The textural properties of the introduced structures, through this study, had been further tracked via determining their surface characteristics. Table 2 summarizes the numerical values of surface parameters for both the as-synthesized polystyrene and its sub-driven ZnNPs@polystyrene composite. It can be observed that the specific surface area and its dependent features (pore volume and pore radius) of polystyrene could be subsequently influenced after loading the ZnNPs. Particularly, the values of both specific surface area and total pore volume of ZnNPs@polystyrene composite had increased nearly to the double, compared to blank polystyrene. These increments can be attributed to the presence of increased weight percent of $\mathrm{Zn}$ particles onto the polystyrene structure (Jassim et al. 2016). On the other hand, the average pore diameter of the composite had declined by $25 \%$, in a comparison to the blank polymer. This decrement may be referred to the smaller pores radii of the platted nanoparticles (Liu 2006) of zinc, which represent $50 \mathrm{Wt}$. \% of the composite, than those of polystyrene. Thus, the average pore diameter of the generated ZnNPs@ polystyrene composite was found to be slightly less than that of the blank polystyrene. Nevertheless, both of the synthesized polystyrene and ZnNPs@polystyrene composite are of mesoporous nature. The 
reasonable specific surface area of the presented ZnNPs@polystyrene composite, as detected, could play a key role during its usage as a corrosion inhibitor.

Figure 4

Table 2

\section{Elemental and Thermal Analyses}

After the structural and textural characteristics of the presented ZnNPs@polystyrene composite had been verified, another confirmation for the presence of $\mathrm{Zn}$ particles, within its structure, had been carried out. Specifically, an elemental analysis test using energetic dispersive X-ray (EDX) technique was performed to the composite sample. In reference to the displayed EDX spectrum in Figure 4e, two highly intense signals assigned for carbon element could be noticed. The increased intensity of C-element peak can strongly confirm the high molecular weight of the polystyrene polymer since that carbon represents a major percent of its content.

Simultaneously, high intensity peaks, indicative to zinc element, could be also noticed in the exhibited spectrum. The detection of such peaks could further confirm the successful incorporation of both polystyrene and $\mathrm{Zn}$ nanoparticles into a composite structure. The elemental analysis could exhibit that $\mathrm{Zn}$ is representing $48 \mathrm{Wt} \%$ of the sample while $\mathrm{C}$ was shown as $50 \mathrm{Wt} \%$. Additionally, two weak signals, referring to both oxygen and sulfur elements, could be observed in the given EDX spectrum. These signals are attributed to the species of initiator (potassium per-sulfate) that could be potentially stuck within the polystyrene structure during its preparation stage. The detection of oxygen indicative peak, through the EDX analysis, may further confirm the existence of hydroxyl groups, due to confinement of water molecules, within the structure of polystyrene, as previously illustrated in FTIR spectra. A side to elemental analysis, a mapping of the prepared ZnNPs@polystyrene composite could be provided via EDX analysis, as illustrated in Figure 4f. In agreement to both SEM and TEM images, ZnNPs could be noticed as highly dispersed and well-distributed in between the $\mathrm{C}$ particles of the polystyrene polymer.

The presence of high percentage of ZnNPs in the ZnNPs@ polystyrene composite had been verified through another tool of analysis, namely; thermal gravimetric analysis (TGA) test. Also, this test was carried out in order to explore the suitability of using the introduced ZnNPs@polystyrene composite as a corrosion inhibitor at high temperature processes. Figure 
5 illustrates the thermal behavior of the synthesized ZnNPs@polystyrene composite through the presented TGA graph. Three stages of weight losses could be noticed showing a total weight loss of $42.30 \%$. The first stage of weight loss could be noted between 0 and $300{ }^{\circ} \mathrm{C}$ in which $5 \mathrm{Wt} \%$ of the sample. Then, another $4.21 \mathrm{Wt} \%$ of the sample was lost as the temperature was increased to $400{ }^{\circ} \mathrm{C}$. These weight losses can be attributed to the release of adsorbed water molecules onto the polymer structure as well as degradation of the terminal carbon skeleton in the aliphatic chain of polystyrene. The former two stages were followed by another stage of massive weight loss, came up to $33 \%$, between 400 and $450{ }^{\circ} \mathrm{C}$. Therefore, a total weight loss of $42.3 \%$ could be observed between 0 and $450{ }^{\circ} \mathrm{C}$.

This high percentage of weight loss can be attributed to the complete decomposition of polystyrene polymer. On the other hand, the remains of the sample, which represents nearly 57 $\%$, could show a steady state of weight between 450 and $700{ }^{\circ} \mathrm{C}$. No detection of weight loss at that temperature range could be explained due the complete domination of ZnNPs which are of a high thermal stability up to $900{ }^{\circ} \mathrm{C}$, as reported in literature (Liu 2006). The displayed data of TGA in Figure 5, could conclude that ZnNPs are presented in the introduced ZnNPs@polystyrene composite by an elevated weight \%. Additionally, ZnNPs@polystyrene composite is highly suitable for using as a corrosion inhibitor in the processes that can hold increased temperature up to $300-350{ }^{\circ} \mathrm{C}$.

Figure 5

\section{Corrosion Inhibition Performance}

By completion of determining structural, textural, elemental and thermal characteristics of the prepared ZnNPs@polystyrene composite, it was then forwarded to the process of corrosion inhibition for carbon steel surfaces. The weight loss technique was utilized in order to study the effect of ZnNPs@polystyrene composite on the corrosion inhibition performance under the influence of various corrosive media. However, before investigating the efficiency of the introduced inhibitor, it was necessary to find out the corrosion percentages of carbon steel surfaces in the absence of the ZnNPs@polystyrene composite. Figure 6a illustrates the detected corrosion percentages for the steel samples during 10 testing days. It can be noticed that the water, as a corrosive media, could show the lowest corrosion percentage. This can be attributed to the slow interaction between the water molecules and the steel metal. Therefore, limited attacks by the corrosive sites (hydroxyl groups) in water molecules toward steel surfaces could undergo (Singh et al. 2014). Hence, a small corrosion percent could be noticed. 
Subsequently, introducing the formation water as a corrosive media could result in a bit higher corrosion percent for the carbon steel sample. This increment can be referred to the presence of larger number of corrosive sites in formation water than that of pure water (Deyab and Abd El-Rehim 2014). Particularly, formation water is normally containing crude oil droplets and mineral salts which can be of strong electron withdrawing behavior. Thus, the corrosion action could be further stimulated, in a comparison to the case of using pure water (Deyab and Abd El-Rehim 2014). On the other hand, the sulfuric acid had displayed the highest corrosion percentages (nearly $25 \%$ ) among the four tested corrosive media. This enhanced percentage of corrosion can be explained due to the chemical composition of sulfuric acid. Chiefly, it contains four oxygen atoms, which are of strong electron withdrawing nature because of their high electron negativity. Therefore, increased probabilities of interactions between these atoms and steel surfaces could take place (Ugi et al. 2016); leading to a quite high corrosion percentage. Similar to sulfuric acid, diesel fuel (as a corrosive media) could arouse a higher corrosion percentage than the two aqueous media, but less than the sulfuric acid. Specifically, diesel fuel could show double and triple the detected corrosion percentages by formation and pure water respectively. The increased corrosive capability of diesel fuel over aqueous media can be referred to the presence of sulfur and aromatic compounds within its composition. These components are of withdrawing nature (Odewunmi et al. 2020), however, with less ability than the corrosive sites in sulfuric acid.

Figure 6

\section{Impact of corrosion inhibitor dose}

In current stage of this research study, the effect of varying the corrosion inhibitor dose on both the corrosion amount (weight loss in grams) and corrosion inhibition efficiency (CIE) was monitored. The collected results through this stage are presented in Table $\mathbf{3}$ and supplementary file (Figure S1). The presented data showed weight loss of carbon steel coupons against time in the aforementioned four corrosive media, for 10 testing days. The corrosion test was carried out in the four different media either in absence or presence of ZnNPs@polystyrene composite, as a corrosion inhibitor. It could be detected that the weight losses of carbon steel coupons increase slightly with time increase in presence of ZnNPs@polystyrene composite. On the other hand, increased amounts of weight loss could be seen in absence of the corrosion inhibitor. This can be explained by the adsorption of ZnNPs@polystyrene composite, as a corrosion inhibitor, onto the carbon steel surface forming a protective layer. This layer could consequently reduce the contact between the metal surface 
and any of corrosive medium. Therefore, the aggressive interaction between the metal surfaces and corrosive media could be minimized (Hegazy 2015).

The detected weight losses and the values of CIE could show that ZnNPs@ polystyrene composite can effectively acts as a good corrosion inhibitor. Additionally, the observations of linear curves (for the solutions which contain ZnNPs@polystyrene composite) fall far below the curves of no inhibitor, as shown in (supplementary file, Figure S1), could strongly verify the high efficiency of the introduced inhibitor. General speaking, the lowest weight loss (in presence of the inhibitor) could be detected, after 10 days of testing, in case of introducing water as a corrosive media. From Table 3, the lowest CIE could be observed by using the inhibitor for a time of 10 days in presence of sulfuric acid, as a corrosive medium. Briefly, it can be depicted that the increment of testing time had been accompanied with increases in values of weight losses. However, these losses had been observably less in presence of ZnNPs@polystyrene composite, as a corrosion inhibitor, than in case of its absence.

The increment in weight losses of carbon steel coupons, by time prolongation, can be attributed to the increased interactions of their surfaces with the corrosive sites in each media. Nevertheless, probabilities of such interactions could be decreased via using ZnNPs@polystyrene composite, as a corrosion inhibitor. The declination in the interactions potentiality between carbon steel surfaces and the corrosive sites can be referred to the formation a barrier layer (by the corrosion inhibitor particles) onto the surface of steel coupons. This in turn could restrict the interactive corrosive sites, in each medium, to strongly reach the surfaces of carbon steel coupons (Aribo et al. 2017). Thus, the attained weight losses by effect of corrosion could be obviously decreased via introducing ZnNPs@polystyrene composite, as an inhibitor, in the current study. Referring to Table 3, the differences in weight losses, after 10 days, in presence of ZnNPs@polystyrene composite and its absence had been less in case of water and formation water than in case of sulfuric acid and diesel fuel media. These differences in weight losses can be referred to the quite aggressive attacks, toward the steel surfaces, by the latter two media than in case of water and formation water. These stronger attacks can be explained by the increased number of corrosive sites in diesel fuel and sulfuric acid compared to those of aqueous media (Muthamma et al. 2021). In an agreement with illustrated data in Table 3, the maximum CIE could be noticed, after 10 days, in case of using pure water. On the other hand, the lowest CIE could be noticed in case of introducing sulfuric acid as a corrosion medium. This percentage (nearly $80 \%$ in case of using 200 ppm as a dose) is, however, still reasonably high in the field of metallic surfaces protection. 
It can be generally noticed that the increment of corrosion inhibitor dose could in turn reduce the corrosion amounts of carbon steel, at a certain time for each. This reduction can be attributed to the increment in number of the corrosion inhibitor particles (Sehmi et al. 2020). Therefore, the probability of interaction between the inhibitor and corrosive media could, on one hand, be enhanced. On the other hand, this increment of particles number could consequently lead to enhanced coverage of steel surface in comparison to at the lower dose. Thus, low corrosion percentages could take place due the limited interaction between the corrosive media and steel surface.

In reference to Table 3 and supplementary file (Figure S1) the corrosion inhibition efficiency had been gradually decreased by time, till the end of testing period, in case of using 400 and 800 ppm as doses of ZnNPs@ polystyrene composite. In contrast, quite sharp declines could be noticed, after 10 days, through applying 200 ppm as an inhibitor dose. This sharp drop could be attributed to the exhaustion of a quite increased number of the inhibitor particles by time passing (Al-Bghdadi et al. 2019). Hence, the remained non-exhausted particles could not potentially provide efficient coverage to the steel surface. For the case of using 400 and 800 ppm, as doses, the decline of inhibition efficiency had lasted as gradual from the beginning of test time to its end. This observation can be explained by the presence of a quite reasonable number of the non-exhausted particles of ZnNPs@polystyrene composite along the testing time. This could subsequently result in minimizing the corrosion amounts since these particles could properly cover the surfaces of steel.

In a conclusion of this stage, it can be stated that the increment of CIE is of a strong dependence to the increment of the inhibitor dose. Additionally, the use of $800 \mathrm{ppm}$ as an inhibitor dose could always achieve efficiencies of higher than $90 \%$ under the effect of the introduced corrosive media. Such percentages can be counted as of a high level in comparison to the usually achieved percentages by the organic inhibitor in thus field.

\section{Effect of temperature}

As soon as the best dose of inhibitor ( $800 \mathrm{ppm})$ was picked, it was implemented at this stage at which impact of different temperatures on the corrosion process had been tracked. Both the weight loss of carbon steel and the corresponding CIEs by ZnNPs@polystyrene composite are illustrated in Table 4 and supplementary file (Figure S2). It can be seen that, 
the elevation of temperature had been continuously accompanied with increment in the corrosion-dependent weight losses. The loss in weight can be attributed to the stimulation of corrosive sites movements by the temperature elevation (Nwanonenyi et al. 2017). Thus, increased levels of attacks by the corrosive sites, in the four corrosive media, could take place. The observed decrement of CIE by temperature elevation, in the presence of ZnNPs@polystyrene composite, could refer to formation of a physical adsorptive film between the composite and the steel surfaces (Hegazy 2015). However, different attitudes could be observed for ZnNPs@polystyrene composite in CIE for the four corrosive media.

Specifically, CIE in pure water had been gradually decreased by raising of temperature (up to 10 days of testing). This non-significant decrement can be explained due to the slow rate of interaction between corrosive sites of water and steel surfaces (Nwanonenyi et al. 2017). Therefore, a limited attack by the stimulated corrosive sites of water (by the increase of temperature) could under take. On the other hand, sharper declines in the CIE could be observed, after 10 days, for both formation water and diesel fuel than that in case of pure water. This notice can be referred to the higher number of motivated corrosive sites, by temperature increase, in the two stated media than in case of pure water. Thus, increased potentialities of attacks by these corrosive sites toward the surfaces of carbon steel could occur. The sharpest decline in the CIE could be noticed by the end of 10 days of test through applying sulfuric acid as a corrosive media. Nevertheless, the attained CIE (nearly $88 \%$ ), after 10 days, at $45^{\circ} \mathrm{C}$ is still considered as quite high in the field of corrosion prevention.

Table 4

\section{Effect of the inhibitor composition}

By completion of the aforementioned stages, it was necessary to find out about the vital role of ZnNPs in inhibiting the corrosion of carbon steel surfaces. Thus, group of ZnNPs@polystyrene composite contain various percentages of ZnNPs had been tested as corrosion inhibitors. In this stage of investigation, the inhibition performances were tested at $25^{\circ} \mathrm{C}$ using sulfuric acid as a corrosive media, an inhibitor dose of $800 \mathrm{ppm}$ and 10 days as a testing time. The obtained CIE by the different composites, which contained ZnNPs ranged between 10 and $50 \mathrm{Wt}$ \%, are displayed through Figure 7a. At first place, the exhibited data could prove that the polystyrene had played a role in inhibiting the corrosion of carbon steel surfaces since an efficiency of $26 \%$ was noted (Layeghi et al. 2016). 
The detected protection of steel surface by the polymer could be explained by the partial coverage of its surface by the polystyrene particles. Therefore, the attacks of corrosive sites toward the steel surface could be particularly reduced. On the other hand, loading $10 \mathrm{Wt}$ \% of ZnNPs onto the polystyrene, forming a composite structure, could significantly enhance the CIE to nearly the double of that the noted efficiency by blank polystyrene. This could obviously reflect the high importance of the present ZnNPs in the composite structure in reducing the corrosion levels. Particularly, the particles of zinc could act as the first defense line against corrosion of carbon steel surfaces during the inhibition process.

Consequently, the increment of zinc weight percentage in the composite structure (corrosion inhibitor) could remarkably enhance CIE. This increment in inhibiting the corrosion can be attributed to the increment in number of zinc nanoparticles by increasing their percentage in the composite. Therefore, the levels of interactions between the particles of corrosion inhibitor and the corrosive sites, in the sulfuric acid, could be elevated (Layeghi et al. 2016). Subsequently, increased percentages of CIE could be detected by the composites that are including higher zinc contents. However, the CIE had been observably increasing by inflation of zinc percentage, in ZnNPs@polystyrene composite, up to $40 \%$. The following increase of zinc particles percentage from 40 to $50 \%$ could slightly increase CIE. This little enhancement of efficiency can be referred to the accumulation of some ZnNPs onto each other by increasing their percentage, in the composite, above $40 \%$. Thus, not all of ZnNPs could be exposed to the corrosive sites in the presented media. Hence, a limited increment in the CIE could be noticed.

Figure 7

\section{Corrosion Tracking}

In the former stages, inhibition efficiency for carbon steel surfaces in different corrosive media using the introduced ZnNPs@polystyrene composite, as a corrosion inhibitor, could be observed via weight loss technique. However, it has been necessary to verify the corrosion inhibition performance through some other analytical tools of analysis. Figure $\mathbf{7 b}$ illustrates the values of contact angle for the surfaces of carbon steel after 10 days of exposure to the different corrosive media (Huhtamäki et al. 2018). The displayed measurements had been taken for the collected steel surfaces after applying an inhibitor dose of $800 \mathrm{ppm}$ at $25^{\circ} \mathrm{C}$ as well as for those steel coupons which had no corrosion inhibitor. 
The noticed values of contact angle have been in a strong agreement with the previously discussed data of corrosion inhibition efficiencies. Particularly, the carbon steel surfaces that had been protected by ZnNPs@polystyrene composite, as an inhibitor, could show higher contact angle values than the steel surfaces which had no protection. The higher is the value of contact angle, the higher is the level of corrosion inhibition. Additionally, the highest contact angle value could be seen for the protected steel surface in the pure water while the lowest one was detected in case of using sulfuric acid, as a corrosive media (Mohamed et al. 2015). These results could prove that the lowest corrosion effect could be attained by the water, as a corrosive media, while the highest level of corrosion could happen in case of sulfuric acid. Further confirmation for the corrosion inhibition efficiency (CIE) for the discussed steel surfaces had been carried out through signifying the charges of their surfaces. Specifically, the values of Zeta potential measurements of these carbon steel samples had been obtained, as displayed in

\section{Table 5.}

The detected negative (-) sign in the shown data via Table 5 could indicate the occurrence of corrosion for the tested carbon steel surfaces. Therefore, the higher is the suffixed numerical value to the sign, the higher is number of negative charges on the steel surfaces. Hence, increased level of corrosions can be assigned for the surfaces which show high negative values (Wei et al. 2018). In reference to the presented data in Table 4, the highest corrosion level could be observed for the carbon steel surface which had been exposed to sulfuric acid in absence of the corrosion inhibitor. On the other hand, the highest corrosion inhibition efficiency can be allocated for the steel surface which was placed in pure water in presence of ZnNPs@polystyrene composite. These findings are strongly matched with the prior acquired data through the weigh losses measurements. The explicited harmony between the results of experimental investigations (via weight loss technique) and those collected by two different analytical tools may strongly verify reliability and consistence of the presented research work.

Table 5

\section{Thermodynamics Parameters}

Activation energy $\left(E_{\mathrm{a}}\right)$ for corrosion of carbon steel surfaces in water, formation water, petroleum diesel fuel and Sulfuric acid in absence and presence of ZnNPs@polystyrene composite (800 ppm), as an inhibitor, at different temperatures $\left(298,308\right.$, and $\left.318^{\circ} \mathrm{K}\right)$ were calculated from Arrhenius equation (1) and its logarithmic form (2): 


$$
\begin{gathered}
r=A e^{(-E a / R T)} \ldots \ldots \ldots \ldots \ldots \ldots \ldots \ldots(1) \\
\ln r=\ln A-\left(E_{a} / R T\right) \ldots \ldots \ldots \ldots \ldots \ldots \ldots \ldots \ldots \ldots \ldots
\end{gathered}
$$

Where, $r$ represents rate of corrosion reaction, $A$ is the Arrhenius constant, $R$ is the gas constant and $T$ is the absolute temperature.

The collected data through the former equations were utilized to produce Arrhenius plots ( $\operatorname{Ln} r$ vs. $1 / T$ ), as displayed in Figure 8. Straight lines with linear regression coefficients very close to 1 could be observed in the four corrosive media. These findings could exhibit that the corrosion of steel surfaces in all the presented media either in presence or absence of ZnNPs@polystyrene composite, as an inhibitor, follow Arrhenius equation with slope of (- $E_{a}$ $/ R$ ) (Refaey et al. 2004). The slopes of the given lines in Figure 8 were then utilized to calculate the activation energies and the other thermodynamic parameters, as listed in Table 5.

\section{Figure 8}

The change in enthalpy and entropy $\left(\Delta H^{*}, \Delta S^{*}\right)$ of activation energy values were calculated from the transition state theory (El-Haddad and Elattar 2013).

$$
\operatorname{Ln}(r / T)=\left[\operatorname{Ln}\left(R / N_{A} h\right)+\left(\Delta S^{*} / R\right)\right]-\left(\Delta H^{*} / R T\right)
$$

Where, $h$ is Plank's constant, $N_{A}$ is the Avogadro's number, $R$ is the ideal gas constant, $\Delta H^{*}$ is the enthalpy of activation and $\triangle S^{*}$ is the entropy of activation.

In reference to Table 6, values of activation energy ( $\left.E_{a}\right)$ for the corrosion of carbon steel surfaces, in different media, have been higher in presence of ZnNPs@polystyrene composite, as an inhibitor, than in case of its absence. These results could indicate that the molecules of ZnNPs@polystyrene composite could attain the inhibition process through a physical adhesion on the steel surface (El-Tabei et al. 2014).

The positive sign of the enthalpy $\left(\Delta H^{*}\right)$ is reflecting to the endothermic nature of the corrosion process. The increased positive values in case of the inhibitor coating than in case of its absence could mean that the dissolution of carbon steel is much difficult in the presence of inhibitor (Refaey et al. 2004). On the other hand, the negative sign of entropy $\left(\Delta S^{*}\right)$ indicates that the activated complex (in rate determining step) represents an association rather than dissociation. This could consequently reflect that the interaction is more taking place between the corrosion media and inhibitor than between the media and carbon steel surface (El-Haddad and Elattar 2013). 


\section{Corrosion Inhibition Mechanism}

Nanocomposites coatings could retard corrosion by more than one action, one of them via playing as the role of barrier as a passive film on steel surface, in addition to the fine dispersion of electrical conductivity inside the polymeric matrix, for efficient corrosion inhibition. The mechanism of the presented ZnNPs@polystyrene composite as a solid corrosion inhibitor, in this research study, could be schematically presented in Figure 9 based on different points of perspectives. The first point is the combination of both a high molecular weight porous polystyrene polymer and metallic zinc nanoparticles. The choice of high molecular weight polystyrene, on one hand, was meant in order to avoid its rapid depletion when being exposed, as a solid dispersant inhibitor, to the different corrosive media.

On the other hand, the choice of porous polymer had been also necessary in order to achieve the highest possible level of zinc nanoparticles impregnation within and onto the polymer geometry. Therefore, a maximum number of accessible zinc nanoparticles can be entrapped within polymeric matrix to produce the desirable composite for sequential functionalization in the corrosion inhibition process. Selection of ZnNPs for preparing the currently required corrosion inhibitor is based on its extensive using in paints since it has high stability onto surfaces.

The second point of perspective in this mechanism had based on the choice of zinc metal which comes, in order, before iron (Fe) in the electrochemical series. This obviously means that zinc particles will be more reactive than iron (steel surfaces) toward the interaction with the corrosive sites in the different media. Thus, increased levels of corrosion inhibition can be effectively achieved. The third point in this mechanism had focused on the enhanced diffusion of ZnNPs@polystyrene composite onto the surface of steel. The high level of ZnNPs@polystyrene composite dispersion onto the surface of steel is assumed to take place through the metal-metal interaction between $\mathrm{Zn}$ particles and Fe surface, providing a physical barrier to obstruct electrolyte permeation. Additionally, the hydrophobicity and aromaticity of the prepared ZnNPs@ polystyrene composite were revealed in higher surface contact angle that can return in higher corrosion inhibition efficiency. Thus, high degrees of corrosion inhibition efficiencies can be strongly achieved. 


\section{Conclusion}

Manufacturing and applications of various organic nanocoatings is progressed towards implementation of organic nanocoatings in many industrial purposes owing to their superior capabilities in different fields. This approach investigated an innovative strategy for synthesis of corrosion inhibitor for steel surfaces based on composite of polystyrene and zinc nanoparticles (ZnNPs@polystyrene composite). The successive immobilization of ZnNPs within polystyrene matrix for clustering of the desirable ZnNPs@polystyrene composite was approved via several instrumental analyses of FT-IR, XRD, SEM, HRSEM, EDX, TEM and BET with estimation of total pore volume and average pore diameter for the prepared composite. Thermal stability of the prepared composite was affirmed via TGA analysis. Corrosion percentage via weight loss percent in three media of water, $\mathrm{H}_{2} \mathrm{SO}_{4}$ and diesel fuel was estimated. Corrosion inhibition efficiency (CIE) percentage estimated according to the weight loss for the steel samples coated with the synthesized ZnNPs@polystyrene composite. Effect of temperature elevation on the estimated CIE after soaking in water, diesel fuel and sulfuric acid as corrosion media was also detected. CIE was estimated for coating steel samples with ZnNPs@polystyrene composite with different doses of ZnNPs. Zeta potential was also detected for untreated and ZnNPs@polystyrene composite treated samples. Eventually, from Arrhenius plots, activation energies and thermodynamic parameters of rate constant, enthalpy $(\Delta H)$, entropy $(\Delta S)$ was also evaluated. Postulation of the reaction mechanism for the anticorrosive action of the synthesized ZnNPs@polystyrene composite was presented according to the illustrated instrumental analyses.

\section{Reference}

Al-Bghdadi SB, Hanoon MM, Odah JF, Shaker LM, Al-Amiery AA Benzylidene as efficient corrosion inhibition of mild steel in acidic solution. In: Multidisciplinary Digital Publishing Institute Proceedings, 2019. vol 1. p 27

Al-Kadhemy MFH, Rasheed ZS (2013) Effect of doping ratio on FTIR spectrum of coumarin doped polystyrene films. Chemistry and Materials Research 3 (12):38-45

Al-Kadhemy MFH, Rasheed ZS, Salim SR (2016) Fourier transform infrared spectroscopy for irradiation coumarin doped polystyrene polymer films by alpha ray. Journal of Radiation Research and Applied Sciences 9 (3):321-331

Aribo S, Olusegun SJ, Ibhadiyi $\sqcup$, Oyetunji A, Folorunso DO (2017) Green inhibitors for corrosion protection in acidizing oilfield environment. Journal of the Association of Arab Universities for Basic and Applied Sciences 24:34-38

Ayeleru OO, Dlova S, Ntuli F, Kupolati WK, Olubambi PA (2019) Development and size distribution of polystyrene/ZnO nanofillers. Procedia Manufacturing 30:194-199 
Calderón J, Jiménez J, Zuleta A (2016) Improvement of the erosion-corrosion resistance of magnesium by electroless $\mathrm{Ni}-\mathrm{P} / \mathrm{Ni}(\mathrm{OH})$ 2-ceramic nanoparticle composite coatings. Surface and Coatings Technology 304:167-178

Cho C, Kwon O, Lee Y (2014) Effects of the sulfur content of liquefied petroleum gas on regulated and unregulated emissions from liquefied petroleum gas vehicle. Fuel 137:328-334

Dariva CG, Galio AF (2014) Corrosion inhibitors-principles, mechanisms and applications. Developments in corrosion protection 16:365-378

Deyab M, Abd El-Rehim S (2014) Effect of succinic acid on carbon steel corrosion in produced water of crude oil. Journal of the Taiwan Institute of Chemical Engineers 45 (3):1065-1072

Du D, Chen K, Lu H, Zhang L, Shi X, Xu X, Andresen PL (2016) Effects of chloride and oxygen on stress corrosion cracking of cold worked $316 / 316 \mathrm{~L}$ austenitic stainless steel in high temperature water. Corrosion Science 110:134-142

El-Haddad MN, Elattar KM (2013) Role of novel oxazocine derivative as corrosion inhibitor for 304 stainless steel in acidic chloride pickling solutions. Research on Chemical Intermediates 39 (7):3135-3149

El-Tabei A, Hegazy M, Bedair A, Sadeq M (2014) Synthesis and inhibition effect of a novel Tri-cationic surfactant on carbon steel corrosion in $0.5 \mathrm{M} \mathrm{H} 2 \mathrm{SO} 4$ solution. Journal of Surfactants and Detergents $17(2): 341-352$

El Naggar AM, Noor El-Din M, Mishrif MR, Nassar IM (2015) Highly efficient nano-structured polymerbased membrane/sorbent for oil adsorption from $\mathrm{O} / \mathrm{W}$ emulsion conducted of petroleum wastewater. Journal of Dispersion Science and Technology 36 (1):118-128

Hegazy M (2015) Novel cationic surfactant based on triazole as a corrosion inhibitor for carbon steel in phosphoric acid produced by dihydrate wet process. Journal of Molecular Liquids 208:227236

Huhtamäki T, Tian X, Korhonen JT, Ras RH (2018) Surface-wetting characterization using contact-angle measurements. Nature protocols 13 (7):1521-1538

Jassim AN, Alwan RM, Kadhim QA, Nsaif AA (2016) Preparation and characterization of $\mathrm{ZnO} /$ polystyrene nanocomposite films using ultrasound irradiation. Nanosci Nanotechnol 6:17-23

Lallam LMOIEmAAMMRSOBOCA (2015) Adsorption and corrosion inhibition of new synthesized Pyridazinium-Based Ionic

Liquid on Carbon steel in $0.5 \mathrm{M} \mathrm{H}$ 2SO4. Journal of Materials and Environmental Science 6 (2):598-606 Lamaka SV, Zheludkevich ML, Yasakau K, Montemor M, Ferreira MG (2007) High effective organic corrosion inhibitors for 2024 aluminium alloy. Electrochimica Acta 52 (25):7231-7247

Layeghi R, Farbodi M, Ghalebsaz-Jeddi N (2016) Preparation of polyaniline-polystyrene-ZnO nanocomposite and characterization of its anti-corrosive performance. International Journal of Nanoscience and Nanotechnology 12 (3):167-174

Liu P (2006) Facile preparation of monodispersed core/shell zinc oxide@ polystyrene (ZnO@ PS) nanoparticles via soapless seeded microemulsion polymerization. Colloids and Surfaces A: Physicochemical and Engineering Aspects 291 (1-3):155-161

Mária TJSTRLP (2014) The use of nano/micro-layers, self-healing and slow release coatings to prevent corrosion and biofouling. Woodhead Publishing Ltd, Cambridge, pp. 135-182. In: Handbook of smart coatings for materials protection. Woodhead Publishing Ltd, Cambridge, pp pp. 135182

Mohamed AM, Abdullah AM, Younan NA (2015) Corrosion behavior of superhydrophobic surfaces: A review. Arabian journal of chemistry 8 (6):749-765

Muthamma K, Kumari P, Lavanya M, Rao SA (2021) Corrosion Inhibition of Mild Steel in Acidic Media by $\mathrm{N}-[(3,4-D i m e t h o x y p h e n y l)$ Methyleneamino]-4-Hydroxy-Benzamide. Journal of Bio-and Tribo-Corrosion 7 (1):1-19

Nwanonenyi S, Ogbobe O, Oguzie E (2017) Protection of mild steel corrosion in sulphuric acid environment using wheat starch. Int J Eng Technol 10:11-21 
Odewunmi NA, Solomon MM, Umoren SA, Ali SA (2020) Comparative Studies of the Corrosion Inhibition Efficacy of a Dicationic Monomer and Its Polymer against API X60 Steel Corrosion in Simulated Acidizing Fluid under Static and Hydrodynamic Conditions. ACS omega 5 (42):27057-27071

Quadri TW, Olasunkanmi LO, Fayemi OE, Solomon MM, Ebenso EE (2017) Zinc oxide nanocomposites of selected polymers: synthesis, characterization, and corrosion inhibition studies on mild steel in $\mathrm{HCl}$ solution. ACS omega 2 (11):8421-8437

Rahmani K, Jadidian R, Haghtalab S (2016) Evaluation of inhibitors and biocides on the corrosion, scaling and biofouling control of carbon steel and copper-nickel alloys in a power plant cooling water system. Desalination 393:174-185

Refaey S, Taha F, Abd El-Malak A (2004) Inhibition of stainless steel pitting corrosion in acidic medium by 2-mercaptobenzoxazole. Applied Surface Science 236 (1-4):175-185

Salehoon E, Ahmadi SJ, Razavi SM, Parvin N (2017) Thermal and corrosion resistance properties of unsaturated polyester/clay nanocomposites and the effect of electron beam irradiation. Polymer Bulletin 74 (5):1629-1647

Sehmi A, Ouici H, Guendouzi A, Ferhat M, Benali O, Boudjellal F (2020) Corrosion Inhibition of Mild Steel by newly Synthesized Pyrazole Carboxamide Derivatives in $\mathrm{HCl}$ Acid Medium: Experimental and Theoretical Studies. Journal of the Electrochemical Society 167 (15):155508

Senthilkumar T, Ajiboye TK (2012) Effect of heat treatment processes on the mechanical properties of medium carbon steel. Journal of Minerals \& Materials Characterization \& Engineering 11 (2):143-152

Singh P, Quraishi M, Ebenso EE (2014) Thiourea-formaldehyde polymer a new and effective corrosion inhibitor for mild steel in hydrochloric acid solution. Int J Electrochem Sci 9:4900-4912

Solomon MM, Gerengi H, Kaya T, Umoren SA (2017) Enhanced corrosion inhibition effect of chitosan for $\mathrm{St} 37$ in $15 \% \mathrm{H} 2 \mathrm{SO} 4$ environment by silver nanoparticles. International journal of biological macromolecules 104:638-649

Tang E, Liu H, Sun L, Zheng E, Cheng G (2007) Fabrication of zinc oxide/poly (styrene) grafted nanocomposite latex and its dispersion. European Polymer Journal 43 (10):4210-4218

Ugi B, Uwah I, Okafor P (2016) Sulphuric acid corrosion of mild steel in leave extracts of Cnidoscolus aconitifolius plant. Chemical and Process Engineering Research 46

Wadi VS, Jena KK, Halique K, Rožič B, Cmok L, Tzitzios V, Alhassan SM (2020) Scalable High Refractive Index polystyrene-sulfur nanocomposites via in situ inverse vulcanization. Scientific reports $10(1): 1-12$

Wang S, Ma Z, Liao Z, Song J, Yang K, Liu W (2015) Study on improved tribological properties by alloying copper to CP-Ti and Ti-6Al-4V alloy. Materials Science and Engineering: C 57:123-132

Wei K, Chen L, Qu Y, Zhang Y, Jin X, Xue W, Zhang J (2018) Zeta potential of microarc oxidation film on zirlo alloy in different aqueous solutions. Corrosion Science 143:129-135

Xu S, Jiao Y, Song N, Li J, Ding CH Preparation and Characterization of Novel Nano-ZnO/Polystyrene Resin Composite. In: Advanced Materials Research, 2012. Trans Tech Publ, pp 220-223 
Figures

[a]

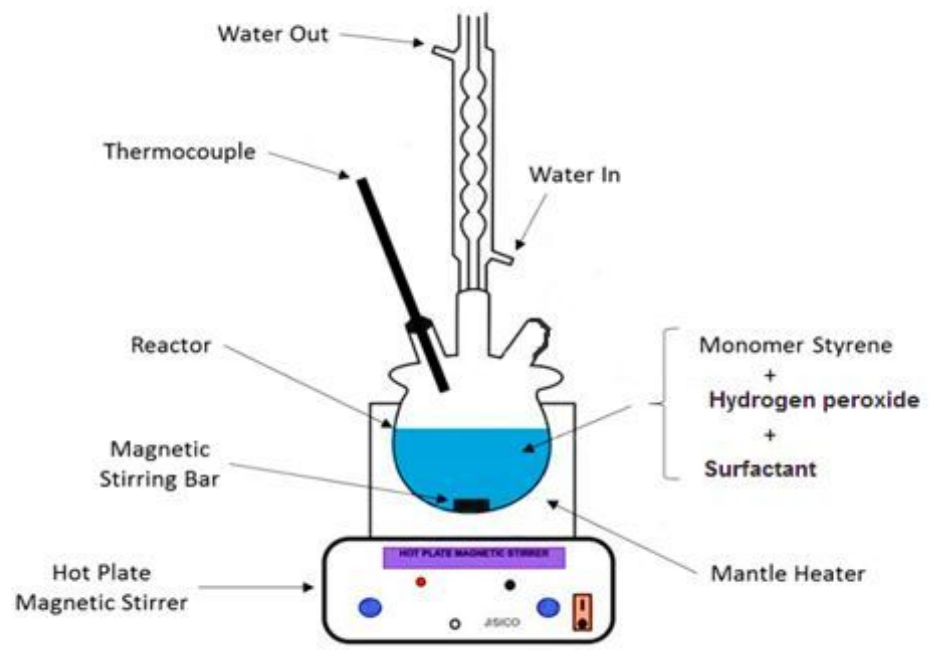

[b] $\quad\left(\mathrm{C}_{2} \mathrm{H}_{5}\right)_{3} \mathrm{~N}$
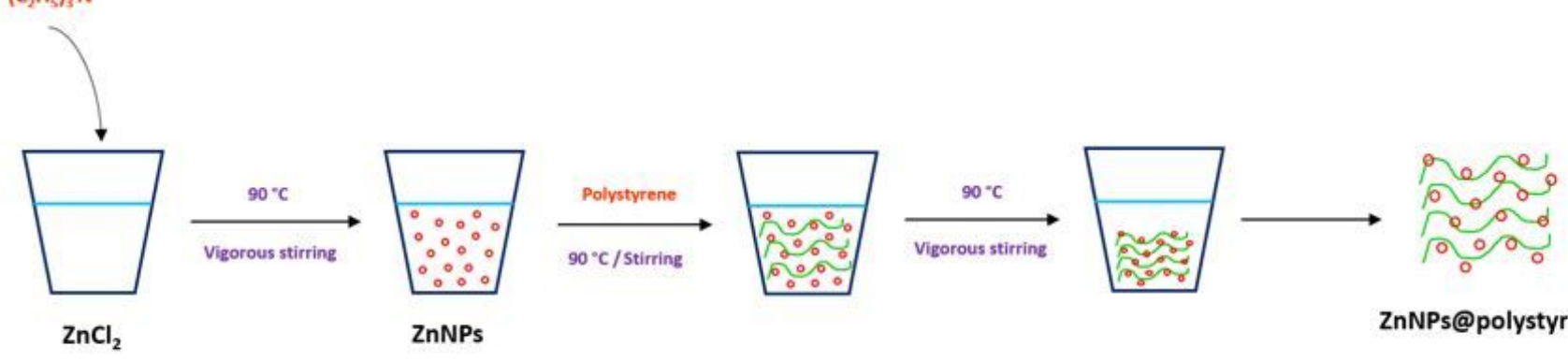

ZnNPs@polystyrene

\section{Figure 1}

Schematic diagram for synthesis; [a] polystyrene and [b] ZnNPs \& ZnNPs@polystyrene composite. 

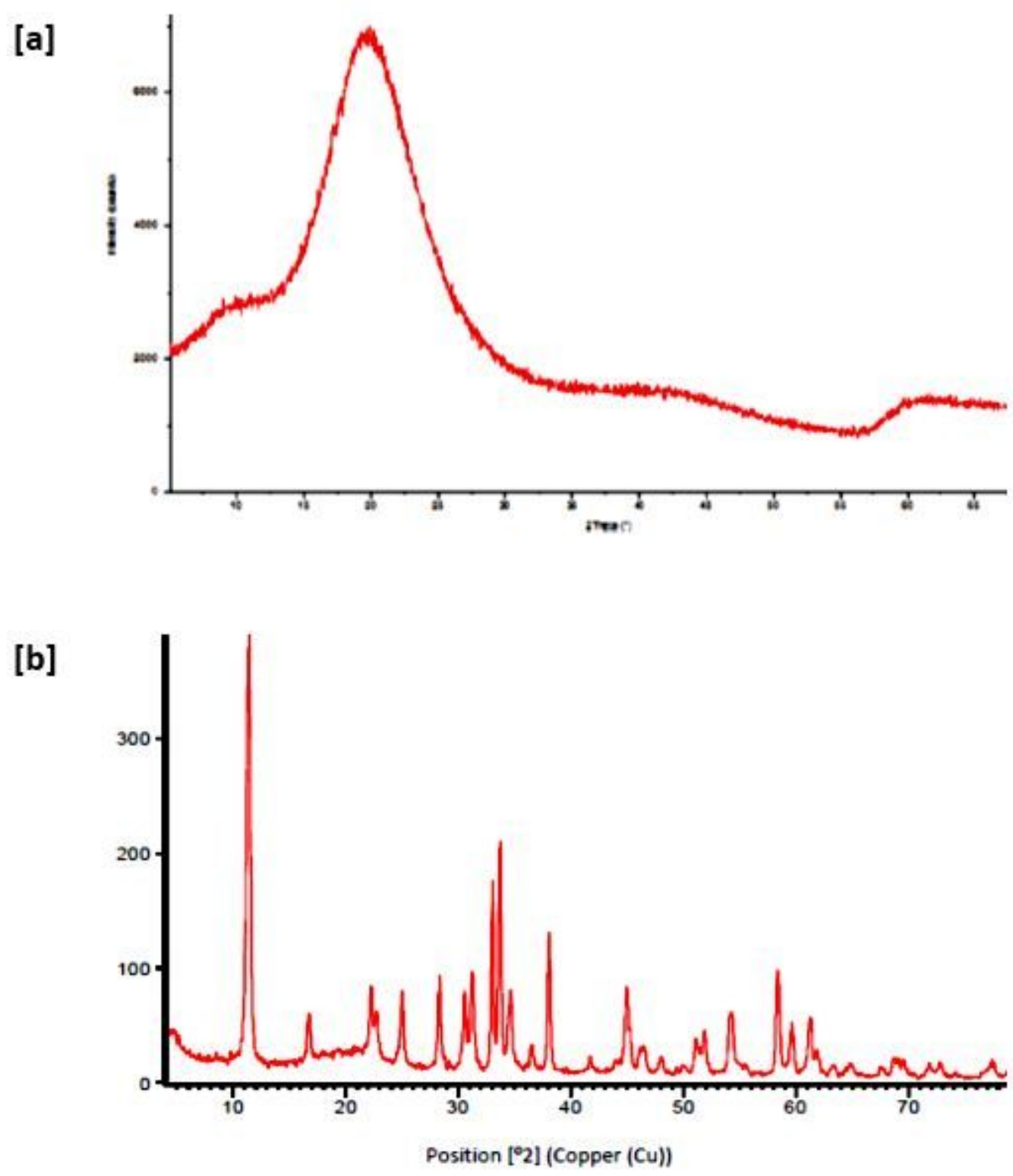

Figure 2

XRD spectra of [a] polystyrene and [b] ZnNPs@polystyrene composite. 
[a]

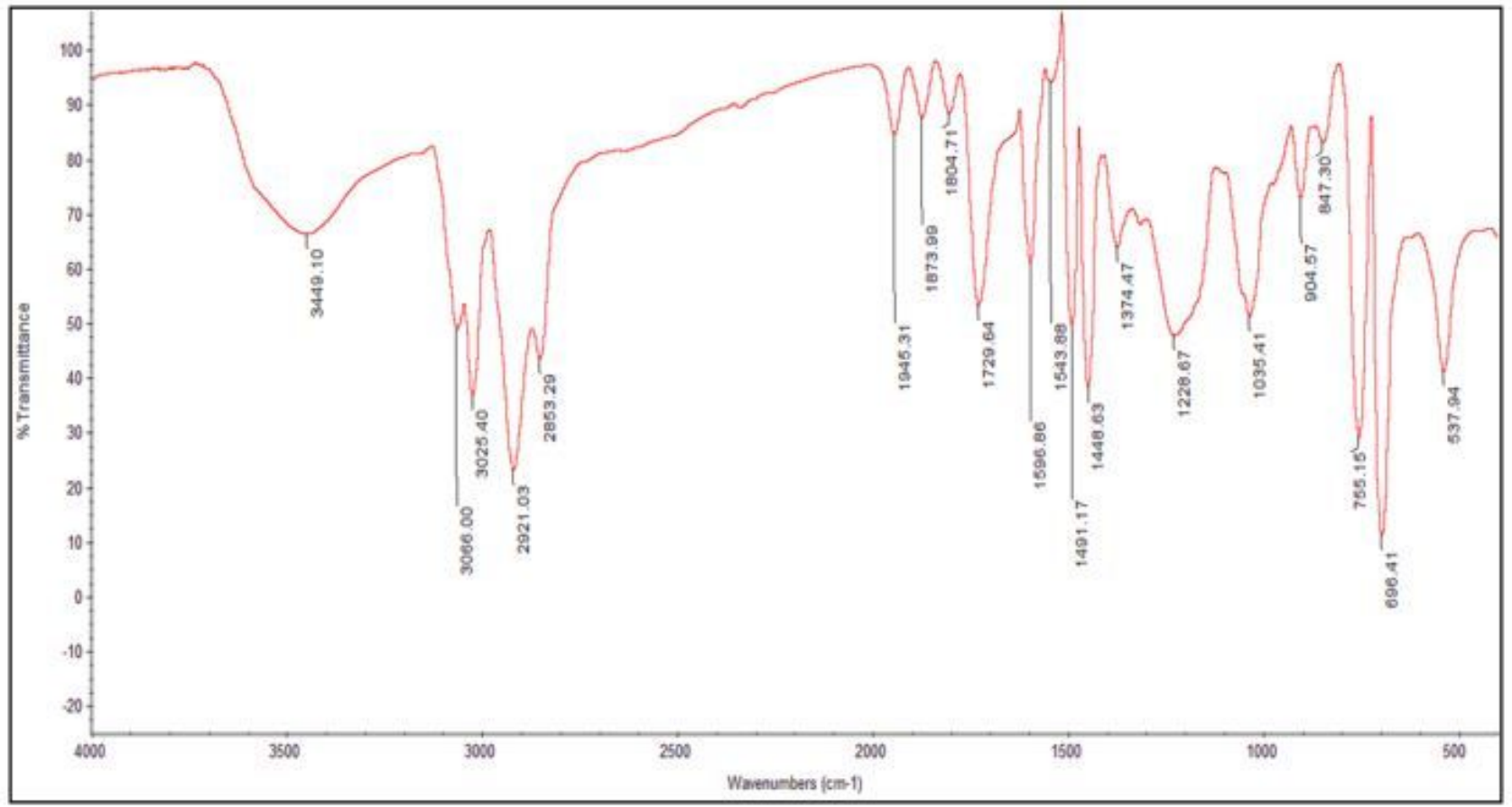

[b]

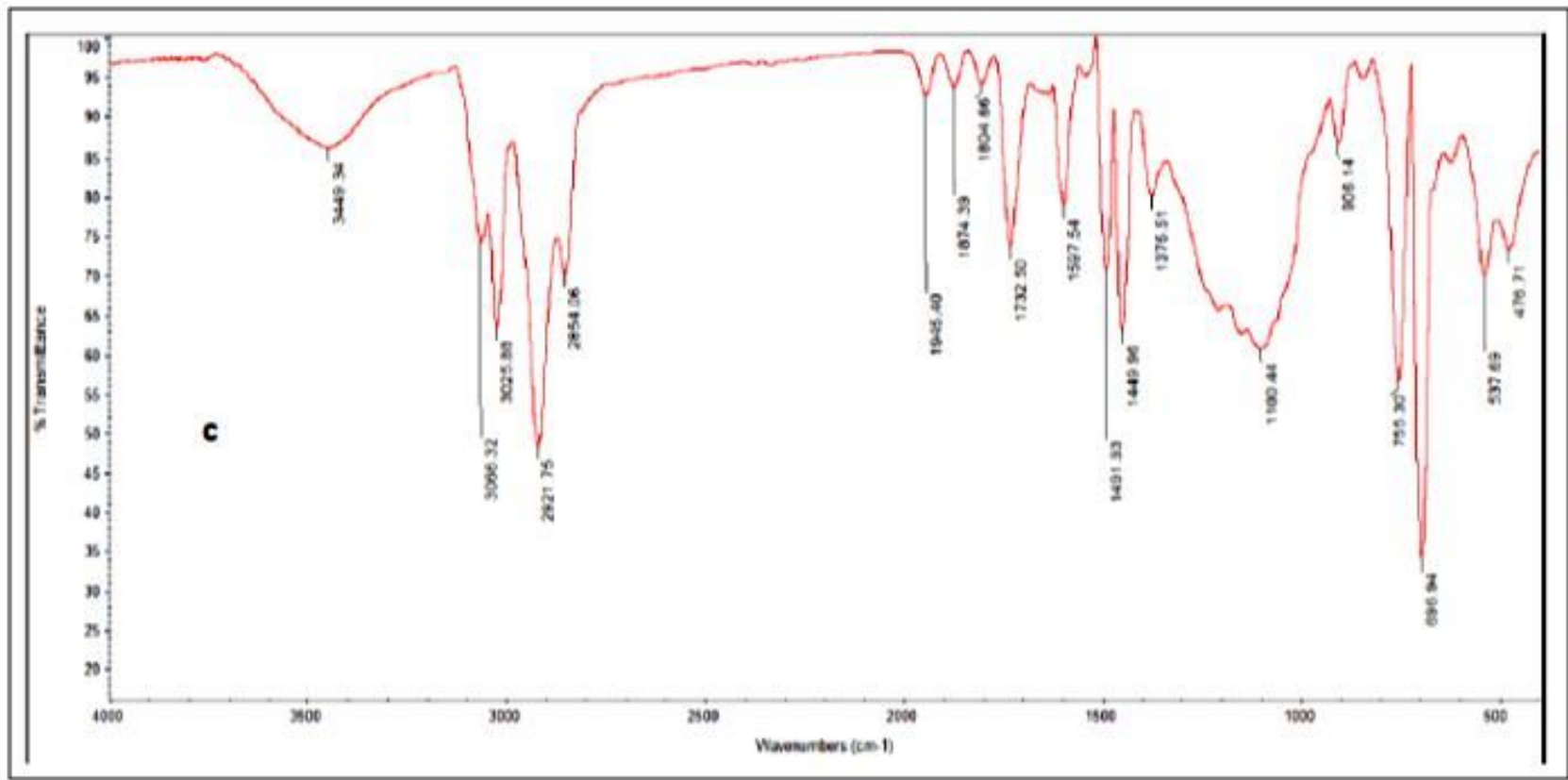

Figure 3

FT-IR spectrum of [a] the as-synthesized polystyrene [b] ZnNPs@polystyrene composite. 
[a]

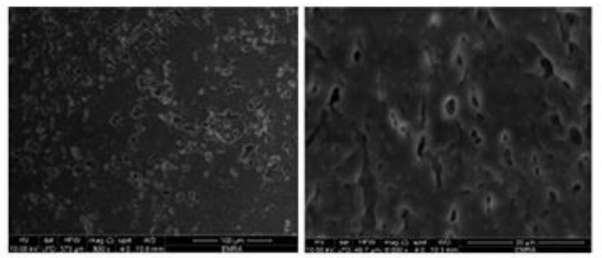

[b]

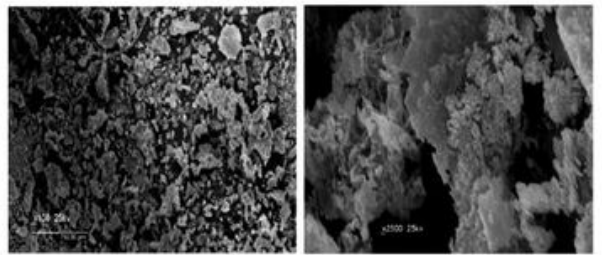

[c]

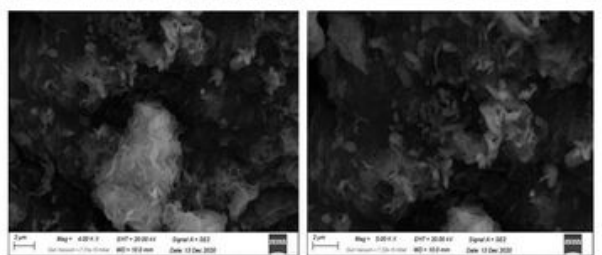

[d]

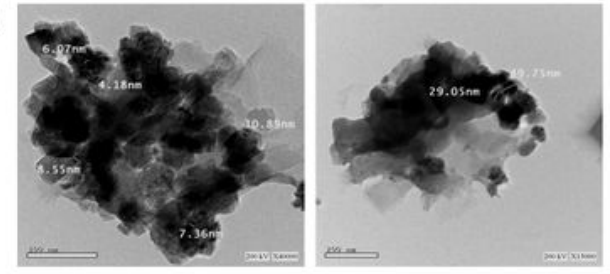

[e]

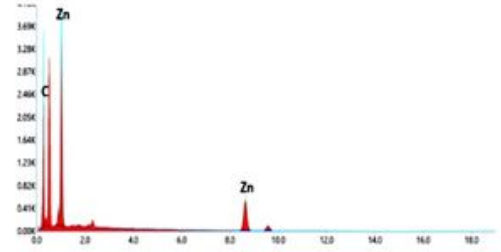

[f]

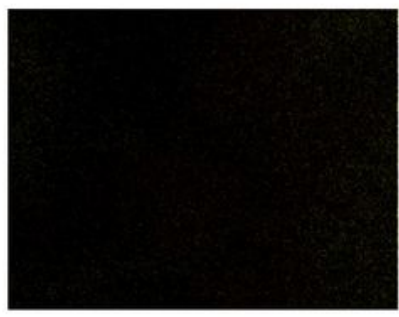

Zn

\section{Figure 4}

Surface morphology of [a] blank polystyrene, [b \& c] the as-prepared ZnNPs@polystyrene composite via SEM \& HR-SEM respectively, [e \& f] EDX spectrum \& mapping of the as-synthesized ZnNPs@polystyrene composite and [d] internal morphology of ZnNPs@polystyrene composite via HRTEM micrographs. 


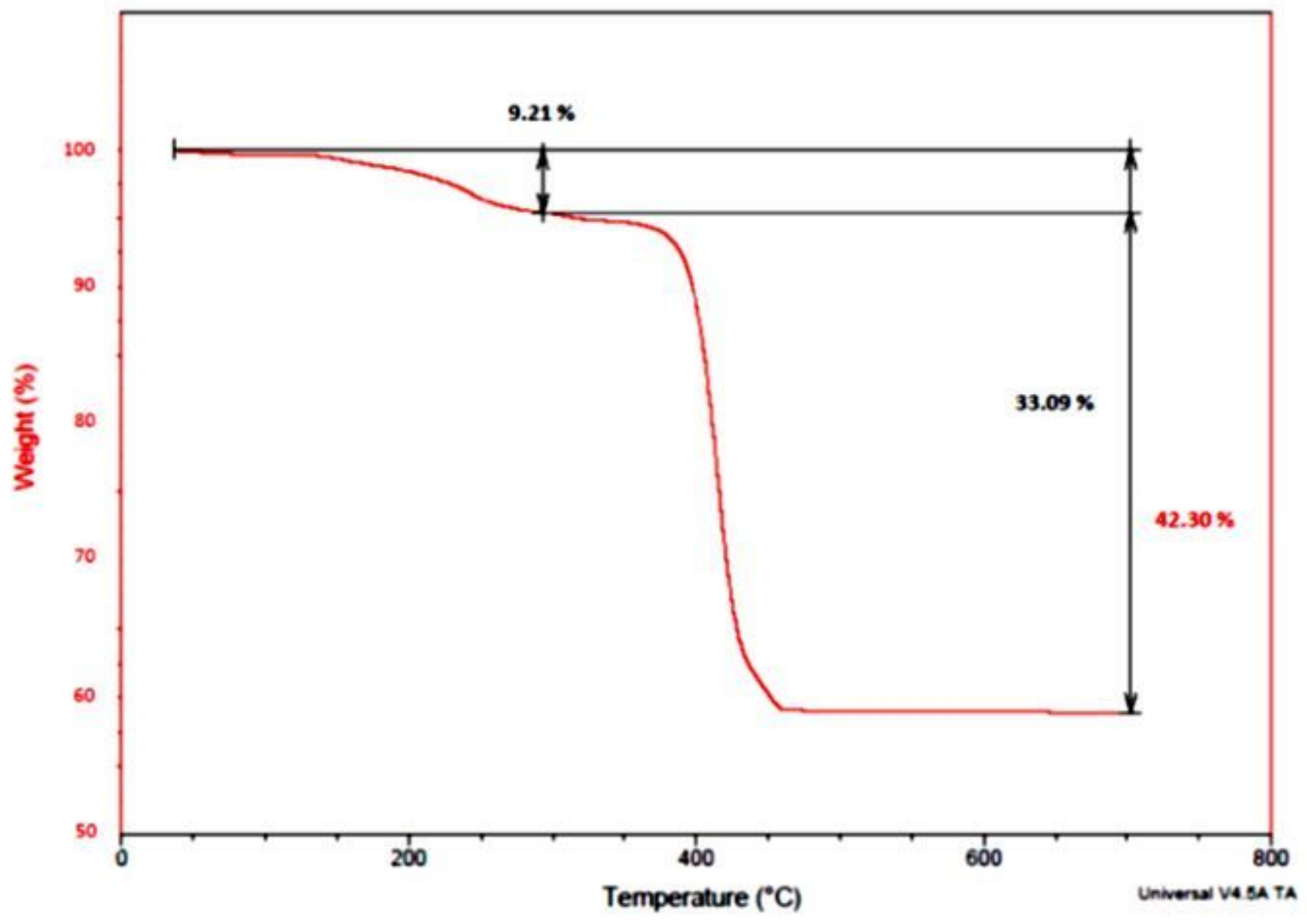

Figure 5

TGA analysis of the as-prepared ZnNPs@polystyrene composite. 


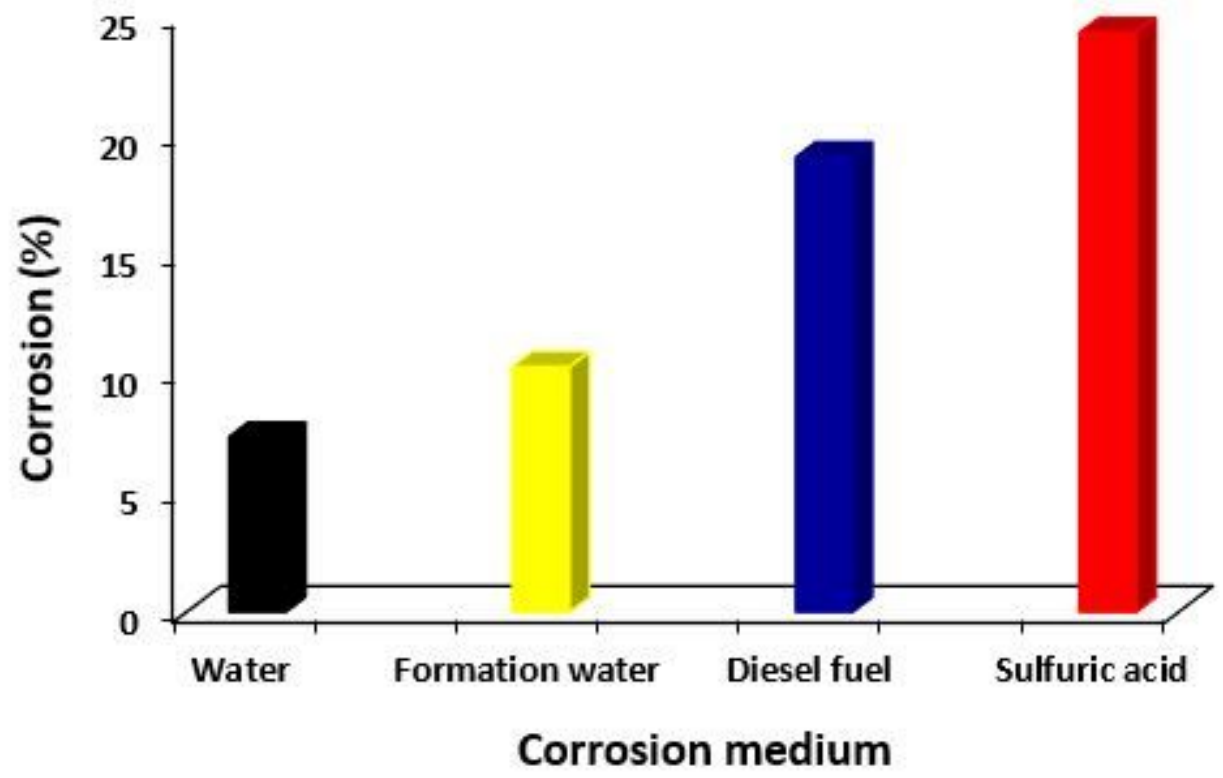

Figure 6

Corrosion percentages of steel metal by effect of different corrosive media (no corrosion inhibitor). 
[a]

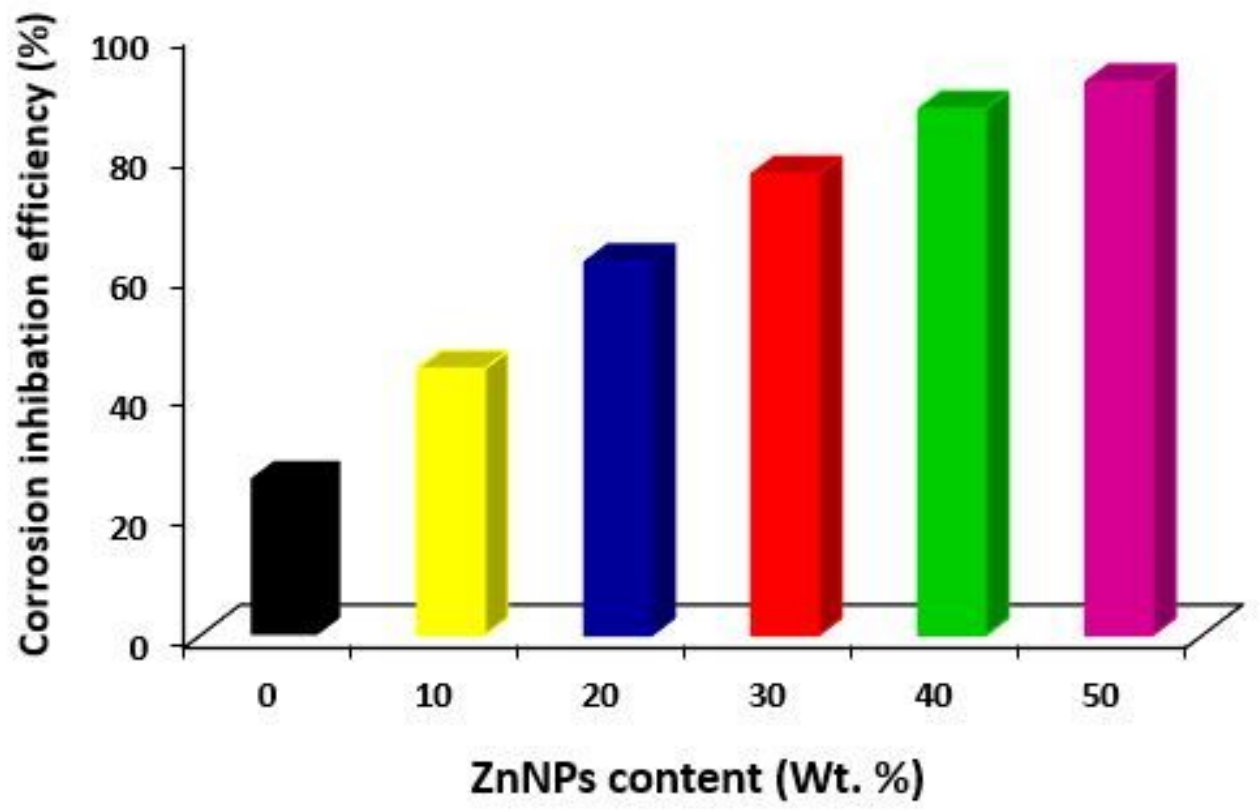

[b]

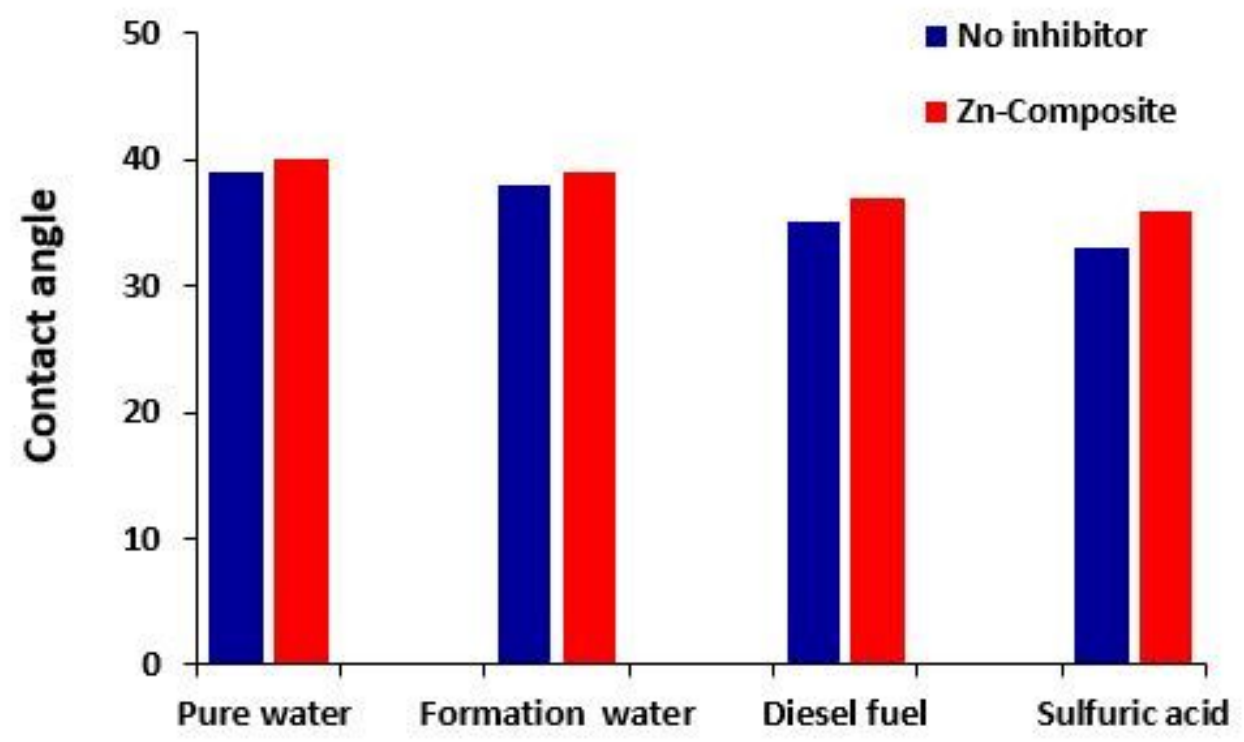

Figure 7

[a] Effect of ZnNPs content on corrosion inhibition efficiency (in sulfuric acid) and [b] contact angle values for the non-protected and inhibitor - protected carbon steel surfaces contact angels. 
[a]

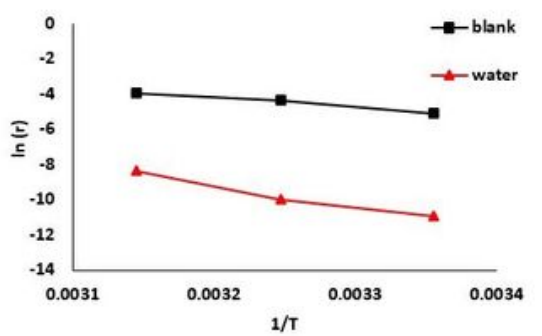

[b]

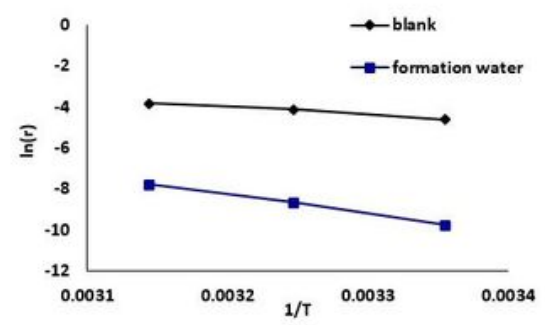

[c]

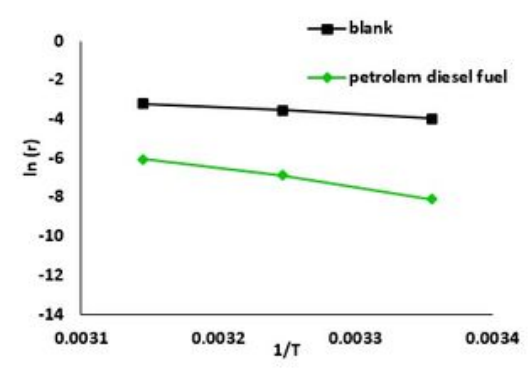

[d]

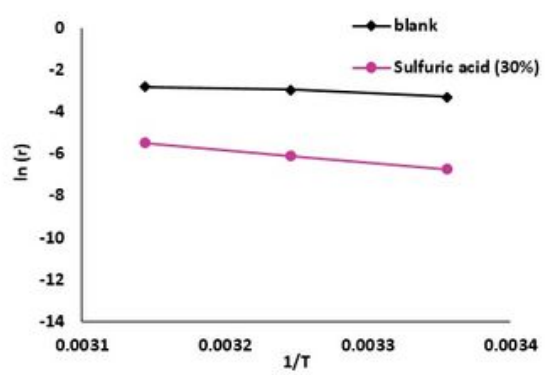

\section{Figure 8}

Arrhenius plots for carbon steel in different corrosive media in absence and presence of the inhibitor; [a] water, [b] formation water, [c] diesel fuel and [d] sulfuric acid. 


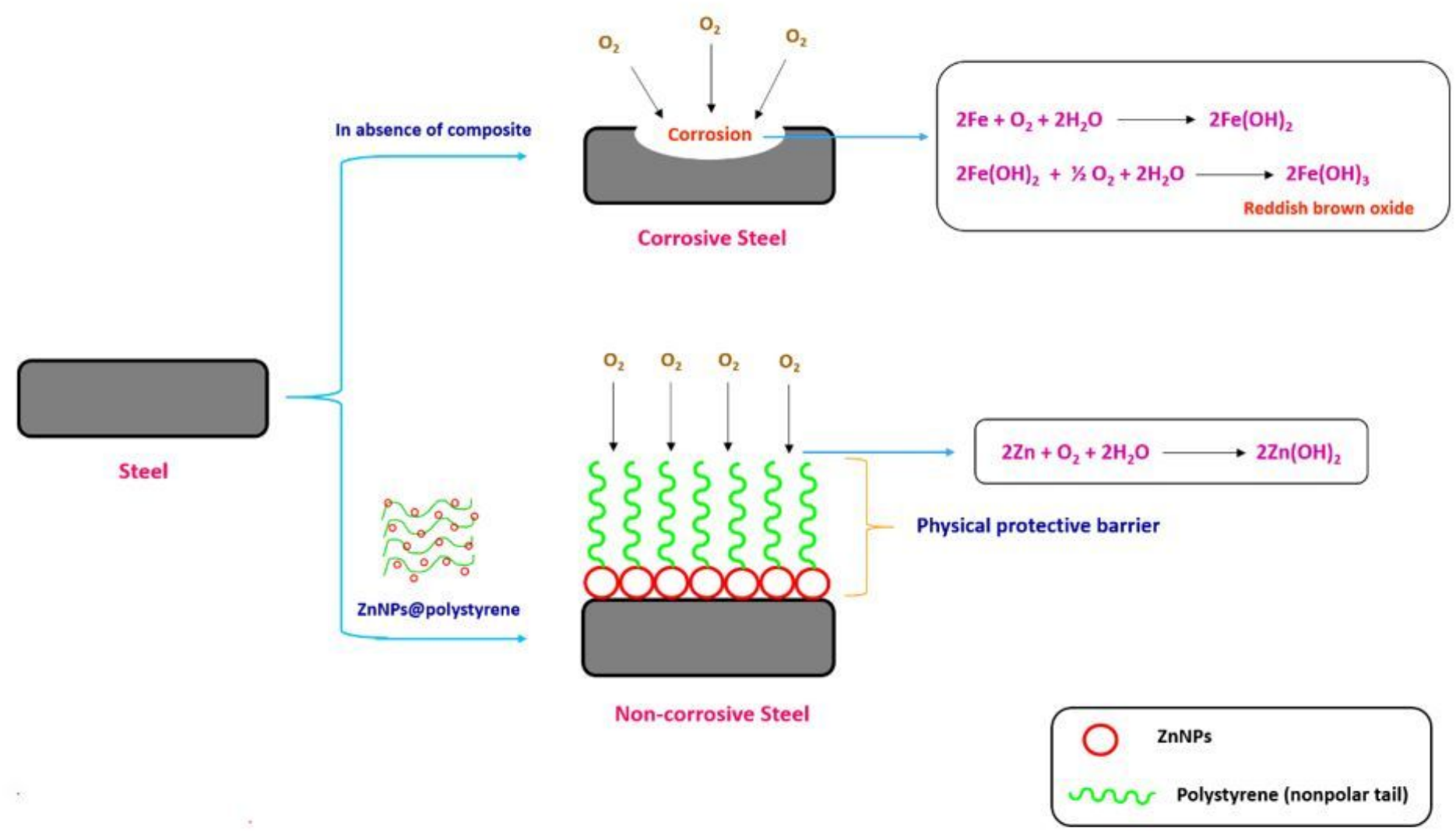

\section{Figure 9}

Schematic diagram for steel surface in absence of anti-corrosive reagent and in the presence of ZnNPs@polystyrene composite as anti-corrosive coating layer.

\section{Supplementary Files}

This is a list of supplementary files associated with this preprint. Click to download.

- SupplementaryFile.docx 